\title{
A Study of the Type II-Plateau Supernova 1999gi and the Distance to its Host Galaxy, NGC 3184
}

\section{Citation}

Leonard, Douglas C., Alexei V. Filippenko, Weidong Li, Thomas Matheson, Robert P. Kirshner, Ryan Chornock, Schuyler D. Van Dyk, et al. 2002. "A Study of the Type II-Plateau Supernova 1999[CLC]gi[/CLC] and the Distance to Its Host Galaxy, NGC 3184." The Astronomical Journal 124 (5): 2490-2505. https://doi.org/10.1086/343771.

\section{Permanent link}

http://nrs.harvard.edu/urn-3:HUL.InstRepos:41417336

\section{Terms of Use}

This article was downloaded from Harvard University's DASH repository, and is made available under the terms and conditions applicable to Other Posted Material, as set forth at http:// nrs.harvard.edu/urn-3:HUL.InstRepos:dash.current.terms-of-use\#LAA

\section{Share Your Story}

The Harvard community has made this article openly available. Please share how this access benefits you. Submit a story.

Accessibility 


\title{
A STUDY OF THE TYPE II-PLATEAU SUPERNOVA 1999gi AND THE DISTANCE TO ITS HOST GALAXY, NGC 3184
}

\author{
Douglas C. Leonard, ${ }^{1}$ Alexei V. Filippenko, ${ }^{2}$ Weidong Li,${ }^{2}$ Thomas Matheson, ${ }^{3}$ Robert P. Kirshner, ${ }^{3}$ \\ Ryan Chornock, ${ }^{2}$ Schuyler D. Van Dyk, ${ }^{4}$ Perry Berlind, ${ }^{3}$ Michael L. Calkins, ${ }^{3}$ Peter M. Challis, ${ }^{3}$ \\ Peter M. Garnavich, ${ }^{3,5}$ Saurabh Jha, ${ }^{3,2}$ and Andisheh Mahdavi ${ }^{3}$ \\ Received 2002 March 20; accepted 2002 July 25
}

\begin{abstract}
We present optical spectra and photometry sampling the first 6 months after discovery of supernova (SN) 1999gi in NGC 3184. SN 1999gi is shown to be a Type II-plateau event with a photometric plateau lasting until about 100 days after discovery. Using the expanding photosphere method (EPM), we derive a distance to SN 1999gi of $11.1_{-1.8}^{+2.0} \mathrm{Mpc}$ and an explosion date of 1999 December $5.8_{-3.1}^{+3.0}$, or $4.1_{-3.1}^{+3.0}$ days prior to discovery. This distance is consistent with a Tully-Fisher distance recently derived to NGC $3184(D \approx 11.59$ $\mathrm{Mpc})$, but it is somewhat closer than the Cepheid distances recently derived to two galaxies that have generally been assumed to be associated with the group containing NGC 3184 (NGC 3319, D=13.30 \pm 0.55 $\mathrm{Mpc}$, and NGC 3198, $D=13.80 \pm 0.51 \mathrm{Mpc}$ ). From many lines of evidence, we conclude that SN 1999gi is only minimally reddened. A comparison between the color of SN 1999gi at early times and that of an infinitely hot blackbody restricts the reddening to $E(B-V)<0.45 \mathrm{mag}$, while a comparison between the color evolution of SN 1999gi and the well-observed (and extensively modeled) Type II-P SN 1999em implies an even more restrictive upper reddening limit of $E(B-V)<0.3$ mag. A variety of other, independent reddening estimates are consistent with these upper limits and yield a probable reddening of $E(B-V)=0.21 \pm 0.09$ mag. We reconsider the upper mass limit $\left(9_{-2}^{+3} M_{\odot}\right)$ recently placed on the progenitor star of SN 1999gi by Smartt et al. in light of these results. Following the same procedures, but using the new data presented here, we arrive at a less restrictive upper mass limit of $15_{-3}^{+5} M_{\odot}$ for the progenitor. The increased upper limit results mainly from the larger distance derived through the EPM than was assumed by the Smartt et al. analyses, which relied on less precise (and less recent) distance measurements to NGC 3184. Finally, we confirm the existence of "complicated" P Cygni line profiles in early-time and later photospheric-phase spectra of SN 1999gi. These features, first identified by Baron et al. in spectra of SN 1999em as high-velocity absorptions, in addition to the "normal" lower velocity component, are here verified to be true P Cygni profiles consisting of both an absorption trough and an emission peak at early times. In the earliest spectrum, taken less than a day after discovery, the features extend out to nearly $-30,000 \mathrm{~km} \mathrm{~s}^{-1}$, indicating the existence of very high velocity material in the outer envelope of SN 1999gi.
\end{abstract}

Key words: distance scale — galaxies: individual (NGC 3184) — supernovae: individual (SN 1999gi)

\section{INTRODUCTION}

Supernova (SN) 1999gi was discovered by Nakano et al. (1999) on 1999 December 9.82 (UT dates are used throughout this paper) at an unfiltered magnitude of $m \approx 14.5$ in the nearly face-on $\left(i<24^{\circ}\right.$, from the Lyon-Meudon Extragalactic Database $[\mathrm{LEDA}]^{6}$ ) SBc galaxy NGC 3184. The identification of hydrogen in an early-time spectrum quickly defined it as a Type II event (Nakano et al. 1999; see Filippenko 1997 for a review of SN types), and the absence of the SN on CCD images of the same field taken 6.64 and 7.32 days earlier (Trondal et al. 1999, limiting unfiltered magnitude 18.5, and Nakano et al. 1999, limiting unfiltered

${ }^{1}$ Five College Astronomy Department, University of Massachusetts, Amherst, MA 01003-9305; leonard@nova.astro.umass.edu.

${ }^{2}$ Department of Astronomy, University of California, Berkeley, Berkeley, CA 94720-3411.

${ }^{3}$ Harvard-Smithsonian Center for Astrophysics, 60 Garden Street, Cambridge, MA 02138.

${ }^{4}$ Infrared Processing and Analysis Center, Mail Stop 100-22, California Institute of Technology, Pasadena, CA 91125.

${ }^{5}$ Current address: Department of Physics, University of Notre Dame, Notre Dame, IN 46556.

${ }^{6}$ At http://leda.univ-lyon1.fr. magnitude 19.0, respectively) implies that it was discovered shortly after explosion.

There have been two previous investigations of $\mathrm{SN}$ 1999gi. In the first Leonard \& Filippenko (2001) examine a single epoch of optical spectropolarimetry of SN 1999gi taken 107 days after discovery. They find an extraordinarily high degree of linear polarization, $p_{\max }=5.8 \%$, where $p_{\max }$ is the highest level of polarization observed in the optical bandpass. If intrinsic to SN 1999gi, such polarization implies an enormous departure from spherical symmetry (Höflich 1991). However, Leonard \& Filippenko (2001) conclude that the majority of the polarization is likely due to interstellar dust and is not intrinsic to the SN. From photometry reported in various IAU Circulars, Leonard \& Filippenko (2001) tentatively classify SN 1999gi as a Type II-plateau supernova (SN II-P). In addition, the total flux spectrum of SN 1999gi from day 107 and a comparable spectral epoch of SN 1999em, a classic SN II-P (Leonard et al. 2002a, hereafter L02; Hamuy et al. 2001) show great spectral similarity, suggesting that they may have been quite similar events.

In the second study Smartt et al. (2001, hereafter S01) examine preexplosion archival Hubble Space Telescope (HST) images of NGC 3184 and use the lack of a 
progenitor-star detection in the prediscovery frame, along with an estimated distance of $D=7.9 \mathrm{Mpc}$, to set an upper limit on the absolute magnitude of the progenitor for SN 1999gi. This is then translated into an upper mass limit of $9_{-2}^{+3} M_{\odot}$ for the progenitor of SN 1999gi through comparison with stellar evolution models. A subsequent reanalysis of the same data by Smartt et al. (2002, hereafter S02), using improved models, confirms this limit. Since stars with initial mass $\lesssim 8 M_{\odot}$ are not expected to undergo core collapse (e.g., Woosley \& Weaver 1986 and references therein), this upper bound sets very tight constraints on the possible mass of the progenitor, a fact that has important implications for the nature of the progenitors of SNe II-P. Indeed, other than the Smartt et al. studies, progenitor masses for $\mathrm{SNe}$ II-P are virtually unconstrained by direct observation. ${ }^{7}$ Although the progenitors of SN 1961V (Goodrich et al. 1989; Filippenko et al. 1995; Van Dyk, Filippenko, \& Li 2002), SN 1978K (Ryder et al. 1993), SN 1987A (e.g., White \& Malin 1987; Walborn et al. 1987), SN 1993J (Filippenko 1993; Aldering, Humphreys, \& Richmond 1994; Cohen, Darling, \& Porter 1995), and SN 1997bs (Van Dyk et al. 1999a) have been identified, all of these $\mathrm{SNe}$ II were peculiar.

In this paper we present 15 optical spectra and 30 photometric epochs of SN 1999gi, sampling the first 169 and 174 days since its discovery, respectively, and derive its distance through the expanding photosphere method (EPM). We present and discuss our photometric and spectroscopic observations in $\S \S 2.1$ and 2.2, respectively. We estimate the reddening of SN 1999gi using a variety of techniques in $\S 2.3$. We apply the EPM to SN 1999gi in $\S 3$ and compare the derived distance with existing estimates of the distance to NGC 3184 in $\S 4.1$. In $\S 4.2$ we discuss the impact of our results on the progenitor mass limits previously determined by $\mathrm{S} 01$ and $\mathrm{S} 02$. We summarize our main conclusions in $\S 5$. Note that much of the background material for the data and analysis presented in this paper, including the details of the photometric and spectral reductions, as well as many specifics of the EPM technique itself, is thoroughly covered by earlier studies and therefore not repeated here. In particular, the recent analysis by L02 of SN $1999 \mathrm{em}$, a strikingly similar event to SN 1999gi, is frequently referenced.

\section{REDUCTIONS AND ANALYSIS}

\subsection{Photometry}

We obtained 30 epochs of BVRI photometry for SN 1999gi, 26 of them taken with the Katzman Automatic Imaging Telescope (KAIT; Filippenko et al. 2001) and four with the $1.2 \mathrm{~m}$ telescope at the Fred Lawrence Whipple Observatory (FLWO). KAIT is equipped with a $512 \times 512$ pixel Apogee CCD camera (AP7) located at the $\mathrm{f} / 8.17$ Cassegrain focus, providing a field of view of $6.7 \times 6.7$ with 0 "! 8 pixel $^{-1}$. The FLWO $1.2 \mathrm{~m}$ is a Ritchey-Chrétien reflector equipped with a 4-Shooter CCD mosaic camera (Szentgyorgyi et al. 2002), consisting of a $2 \times 2$ array of thinned, backsideilluminated, antireflection-coated Loral $2048 \times 2048$ pixel CCD detectors situated at the $\mathrm{f} / 8$ Cassegrain focus, which provides a field of view $11 ! 4 \times 11 ! 4$ on each chip. All expo-

\footnotetext{
${ }^{7}$ Mass constraints on the progenitors of other types of core-collapse $\mathrm{SNe}$ have been obtained through studies of their environments; see, e.g., Van Dyk et al. (1999b).
}

sures with the mosaic were made on the same CCD (chip 3), and our observations were taken in a $2 \times 2$ binned mode, so that the resulting images were sampled at $0.67 \mathrm{pixel}^{-1}$. The seeing, estimated by the FWHM of stars on the CCD frame, generally ranged from 2 ".5 to 4.5 at KAIT and between 1 ".5 to 2".5 at FLWO. Exposure times of 3 to 5 minutes were typical at KAIT and 1 to 3 minutes at FLWO, with the longest exposures taken in $B$.

Figure 1 shows a KAIT $B$-band image of NGC 3184 taken on 1999 December 17. The four "local standards" identified in the field of SN 1999gi were used to measure the relative SN brightness on nonphotometric nights. We reduced the data according to the procedure detailed by L02, except that, instead of using point-spread function (PSF) fitting to measure the SN's brightness, we employed the more accurate technique of galaxy subtraction (e.g., Filippenko et al. 1986; Richmond et al. 1995). To use this technique, an image of the host galaxy without the SN (i.e., a "template" image of the galaxy taken either before the explosion or after the $\mathrm{SN}$ has faded beyond detection) is subtracted from the images containing the SN, with special care taken to match the alignment, intensity, and PSFs of the images with and without the SN present. Since we did not possess deep filtered preexplosion images of NGC 3184, we obtained the templates on 2002 January 17 with the Nickel $1 \mathrm{~m}$ reflector at Lick Observatory. The Nickel telescope utilizes a Loral $2048 \times 2048$ pixel CCD, which we binned $2 \times 2$ to yield a plate scale of 0 ". 37 pixel $^{-1}$.

The absolute calibration of the field was accomplished on the three photometric nights of 2000 April 3 (with KAIT) and 2002 January 17 and 18 (with the Nickel $1 \mathrm{~m}$ ) by observing several fields of Landolt (1992) standards over a range of air masses, in addition to the SN 1999gi field. The calibration of two of the local standards was further verified against the photometric calibrations by S. Benetti (2002, private communication; star A in Fig. 1) and B. Skiff ${ }^{8}$ (star $\mathrm{C}$ in Fig. 1) and found to agree to within the reported uncertainties. We list the measured $B V R I$ magnitudes and the $1 \sigma$ uncertainty (quadrature sum of the photometric error and the $1 \sigma$ scatter of the individual photometric measurements taken on the three nights) of the local standard stars in Table 1.

The transformation coefficients for the KAIT data to the standard Johnson-Cousins (Johnson et al. 1966 for $B V$; Cousins 1981 for $R I$ ) systems are those of L02. For the FLWO data with chip 3 of the 4-Shooter we derived color terms from five photometric nights (1999 December 9, 2000 January 3, January 5, February 7, and February 16) of

$$
\begin{aligned}
& B=b+0.040(B-V)+C_{B}, \\
& V=v-0.045(B-V)+C_{V}, \\
& R=r-0.080(V-R)+C_{R}, \\
& I=i+0.028(V-I)+C_{I},
\end{aligned}
$$

where bvri are the instrumental and $B V R I$ the standard Johnson-Cousins magnitudes. The terms $C_{B}, C_{V}, C_{R}$, and $C_{I}$ are the differences between the zero points of the instrumental and standard magnitudes, determined for each observation by measuring the offset between the instrumen-

\footnotetext{
${ }^{8}$ At http://www.kusastro.kyoto-u.ac.jp/vsnet/Mail/vsnet-chat/ msg02439.html.
} 


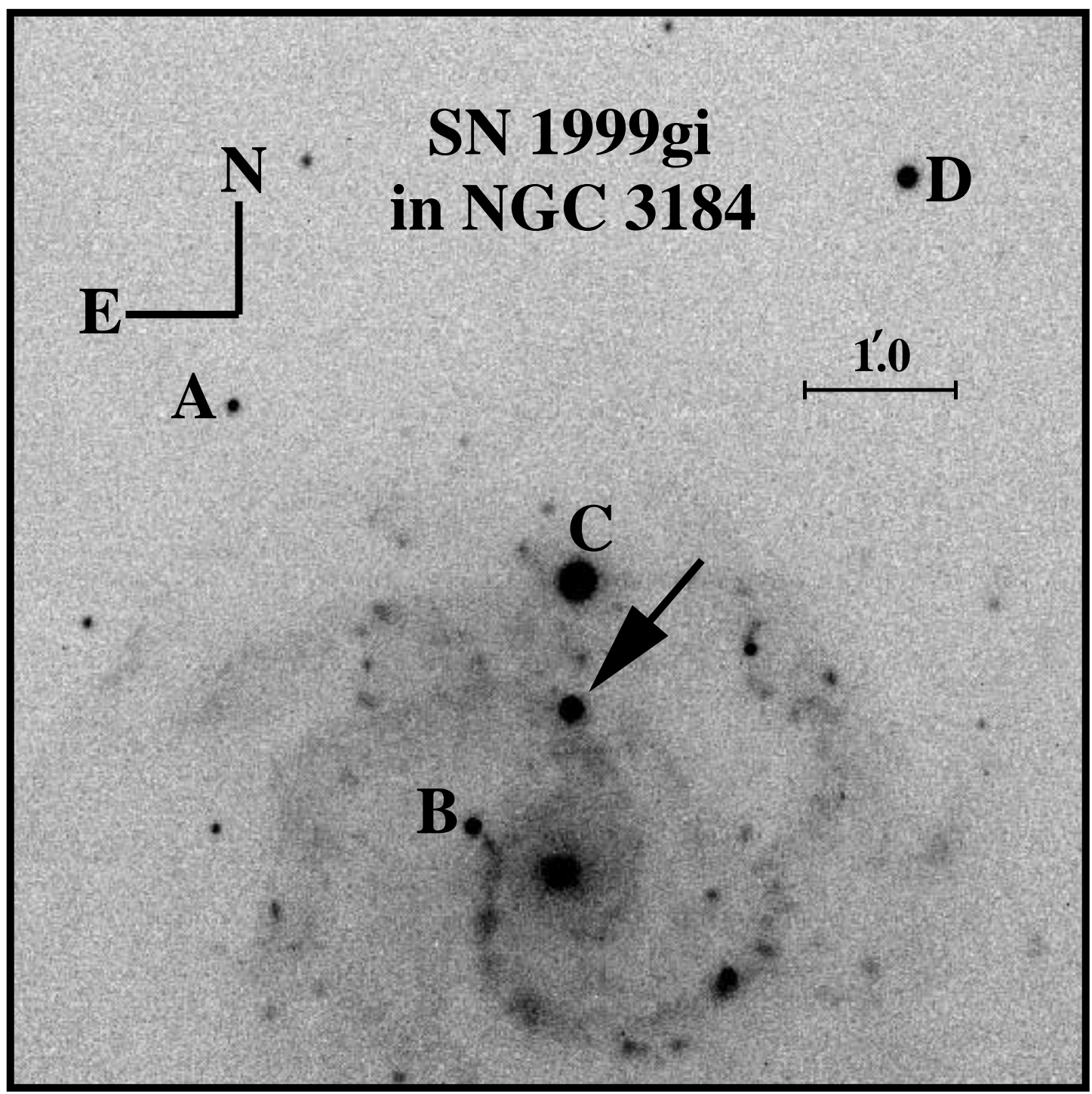

FIG. 1. - B-band image of NGC 3184 taken on 1999 December 17 with the Katzman Automatic Imaging Telescope (Filippenko et al. 2001), with the local standards listed in Table 1 marked. SN 1999gi (arrow) is measured to be 60".9 north and 4".7 west of the center of NGC 3184.

tal and standard magnitudes and colors of the local standard stars.

To transform the photometry to the standard JohnsonCousins system, we took the weighted mean of the values individually derived from the four calibrator stars. Since star C was saturated in nearly all of the FLWO observations, the FLWO photometry was usually derived using only three stars. The results of our photometric observations are given in Table 2 and shown in Figure 2. The reported uncertainties come from the photometric and

TABLE 1

Magnitudes of Local Standards

\begin{tabular}{lcccc}
\hline \hline Star & $B\left(\sigma_{B}\right)$ & $V\left(\sigma_{V}\right)$ & $R\left(\sigma_{R}\right)$ & $I\left(\sigma_{I}\right)$ \\
\hline A ...... & $17.28(0.04)$ & $16.44(0.03)$ & $15.96(0.05)$ & $15.53(0.06)$ \\
B ...... & $16.17(0.04)$ & $15.46(0.03)$ & $15.08(0.04)$ & $14.71(0.04)$ \\
C ...... & $12.56(0.03)$ & $11.97(0.02)$ & $11.64(0.03)$ & $11.33(0.03)$ \\
D ...... & $15.05(0.05)$ & $14.29(0.03)$ & $13.85(0.04)$ & $13.45(0.04)$ \\
\hline
\end{tabular}

transformation errors added in quadrature; in most cases the total error was dominated by the uncertainty in the transformation.

The light curves of SN 1999gi indicate a fairly rapid initial rise followed by a clear plateau of nearly constant brightness in VRI that lasts until about 100 days after discovery. To estimate the date of maximum in the $B$ and $V$ bandpasses (maximum brightness in $R$ and $I$ is not sharply defined and actually comes significantly after the initial rise; see Fig. 3 for detail of the light curves near maximum light), we fitted spline functions to the data around the peak. To estimate the uncertainty in the date of maximum we measured the $1 \sigma$ spread in the derived dates of maximum for 1000 sets of synthetic photometric data, in which each epoch's photometry near the peak was replaced with a value randomly chosen from a Gaussian-weighted distribution centered on the values given in Table 2 and characterized by a standard deviation equal to the uncertainty listed in Table 2 . From this analysis we conclude that $m_{B}(\max ) \approx 14.8$ mag occurred on HJD 2,451,526.2 \pm 1.8 (1999 December $13.7 \pm 1.8$ ), which is $3.9 \pm 1.8$ days after discovery. For $V$ we find 
TABLE 2

Photometric Observations of SN 1999gi

\begin{tabular}{|c|c|c|c|c|c|}
\hline UT Date $^{\mathrm{a}}$ & Day $^{b}$ & $B\left(\sigma_{B}\right)$ & $V\left(\sigma_{V}\right)$ & $R\left(\sigma_{R}\right)$ & $I\left(\sigma_{I}\right)$ \\
\hline $1999-12-10^{c} \ldots \ldots \ldots \ldots . . .$. & 0.62 & $14.950(0.019)$ & $14.986(0.015)$ & $14.894(0.013)$ & $14.784(0.021)$ \\
\hline 1999-12-11 ............. & 1.70 & & $14.865(0.048)$ & $14.603(0.045)$ & $14.441(0.045)$ \\
\hline 1999-12-12 „.............. & 2.74 & $14.873(0.045)$ & $14.784(0.016)$ & $14.475(0.033)$ & $14.338(0.041)$ \\
\hline $1999-12-15 \ldots$. & 5.66 & $14.868(0.031)$ & $14.642(0.021)$ & $14.337(0.025)$ & $14.150(0.023)$ \\
\hline 1999-12-16 „............. & 6.67 & $14.866(0.029)$ & $14.635(0.021)$ & $14.326(0.023)$ & $14.128(0.026)$ \\
\hline $1999-12-17 \ldots$. & 7.65 & $14.876(0.033)$ & $14.626(0.023)$ & $14.300(0.035)$ & $14.119(0.029)$ \\
\hline 1999-12-18 „............. & 8.69 & $14.919(0.051)$ & $14.638(0.035)$ & $14.307(0.038)$ & $14.125(0.033)$ \\
\hline 1999-12-21 „............. & 11.63 & $14.978(0.041)$ & $14.664(0.037)$ & $14.289(0.033)$ & $14.102(0.031)$ \\
\hline 1999-12-27 ...... & 17.66 & $15.177(0.022)$ & $14.683(0.019)$ & $14.241(0.028)$ & $14.007(0.024)$ \\
\hline 2000-01-01 ...... & 22.68 & $15.454(0.022)$ & $14.741(0.018)$ & $14.289(0.031)$ & $14.004(0.047)$ \\
\hline 2000-01-06 ..... & 27.64 & $15.683(0.025)$ & $14.784(0.029)$ & $14.348(0.021)$ & $14.044(0.022)$ \\
\hline $2000-01-12 \ldots$ & 33.61 & $15.856(0.021)$ & $14.841(0.035)$ & $14.365(0.032)$ & $14.058(0.035)$ \\
\hline 2000-02-02 ........ & 54.67 & $16.300(0.031)$ & $14.948(0.039)$ & $14.375(0.045)$ & $13.949(0.051)$ \\
\hline 2000-02-07 .............. & 59.51 & $16.342(0.033)$ & $14.979(0.028)$ & $14.392(0.025)$ & $13.957(0.025)$ \\
\hline 2000-02-15 ............... & 67.61 & $16.447(0.057)$ & $14.982(0.055)$ & $14.384(0.058)$ & $13.968(0.061)$ \\
\hline $2000-02-15^{\mathrm{c}} \ldots \ldots$ & 67.61 & $16.442(0.023)$ & $15.021(0.029)$ & $14.428(0.031)$ & $13.965(0.053)$ \\
\hline $2000-03-10 \ldots$. & 91.53 & $16.603(0.048)$ & $15.114(0.025)$ & $14.464(0.033)$ & $14.036(0.031)$ \\
\hline 2000-03-15 ............... & 96.41 & $16.754(0.040)$ & $15.146(0.024)$ & $14.503(0.027)$ & $14.074(0.030)$ \\
\hline 2000-03-20 .............. & 101.49 & $16.715(0.023)$ & $15.207(0.040)$ & $14.532(0.039)$ & $14.099(0.034)$ \\
\hline 2000-03-25 .............. & 106.45 & $16.917(0.046)$ & $15.330(0.029)$ & $14.642(0.029)$ & $14.189(0.033)$ \\
\hline 2000-03-30 ............. & 111.43 & $17.162(0.026)$ & $15.475(0.032)$ & $14.755(0.032)$ & $14.287(0.033)$ \\
\hline 2000-04-03 „............. & 115.54 & $17.399(0.030)$ & $15.645(0.024)$ & $14.924(0.029)$ & $14.461(0.028)$ \\
\hline 2000-04-08 „............... & 120.43 & $17.818(0.051)$ & $16.099(0.033)$ & $15.233(0.034)$ & $14.729(0.029)$ \\
\hline 2000-04-13 „............. & 125.36 & $18.138(0.024)$ & $16.750(0.066)$ & $15.788(0.153)$ & $15.158(0.047)$ \\
\hline 2000-04-18 „............. & 130.40 & $19.180(0.047)$ & $17.142(0.045)$ & $16.047(0.073)$ & $15.478(0.070)$ \\
\hline 2000-04-29 „............ & 141.37 & $19.274(0.050)$ & $17.436(0.038)$ & $16.285(0.029)$ & $15.689(0.084)$ \\
\hline 2000-05-04 „............. & 146.36 & $19.355(0.059)$ & $17.470(0.040)$ & $16.332(0.042)$ & $15.711(0.040)$ \\
\hline $2000-05-06^{\mathrm{c}} \ldots \ldots \ldots \ldots . .$. & 148.44 & $19.458(0.032)$ & $17.647(0.031)$ & $16.321(0.035)$ & $15.828(0.043)$ \\
\hline 2000-05-12 „............. & 154.36 & $19.430(0.080)$ & $17.590(0.054)$ & $16.417(0.066)$ & $15.781(0.054)$ \\
\hline $2000-06-01^{\mathrm{c}} \ldots \ldots \ldots \ldots \ldots$ & 174.34 & $19.670(0.039)$ & $17.863(0.026)$ & $16.575(0.046)$ & $16.197(0.032)$ \\
\hline
\end{tabular}

\footnotetext{
a yyyy-mm-dd.

b Days since discovery, 1999-12-09.82 UT (HJD 2,451,522.32).

c Photometric observations taken with the $1.2 \mathrm{~m}$ telescope at the Fred Lawrence Whipple Observatory; all others were made with the Katzman Automatic Imaging Telescope.
}

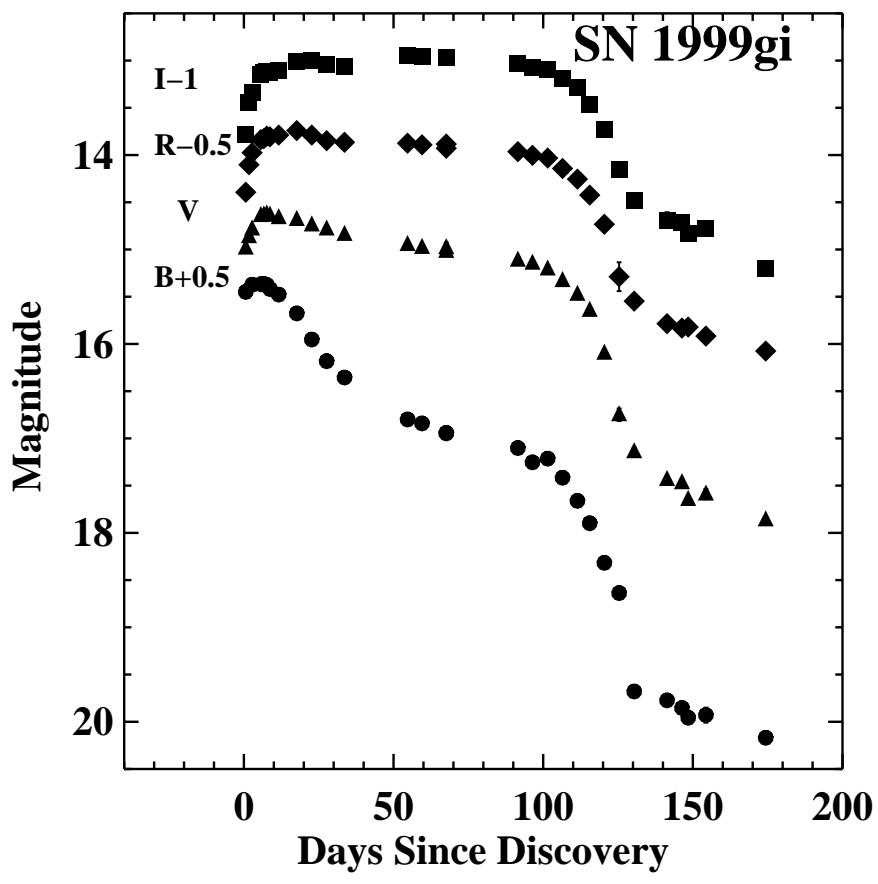

FIG. 2.-BVRI light curves for SN 1999gi from Table 2. For clarity, the magnitude scales for $B R I$ have been shifted by the amounts indicated. In most cases the error bar is smaller than the plotted symbol.

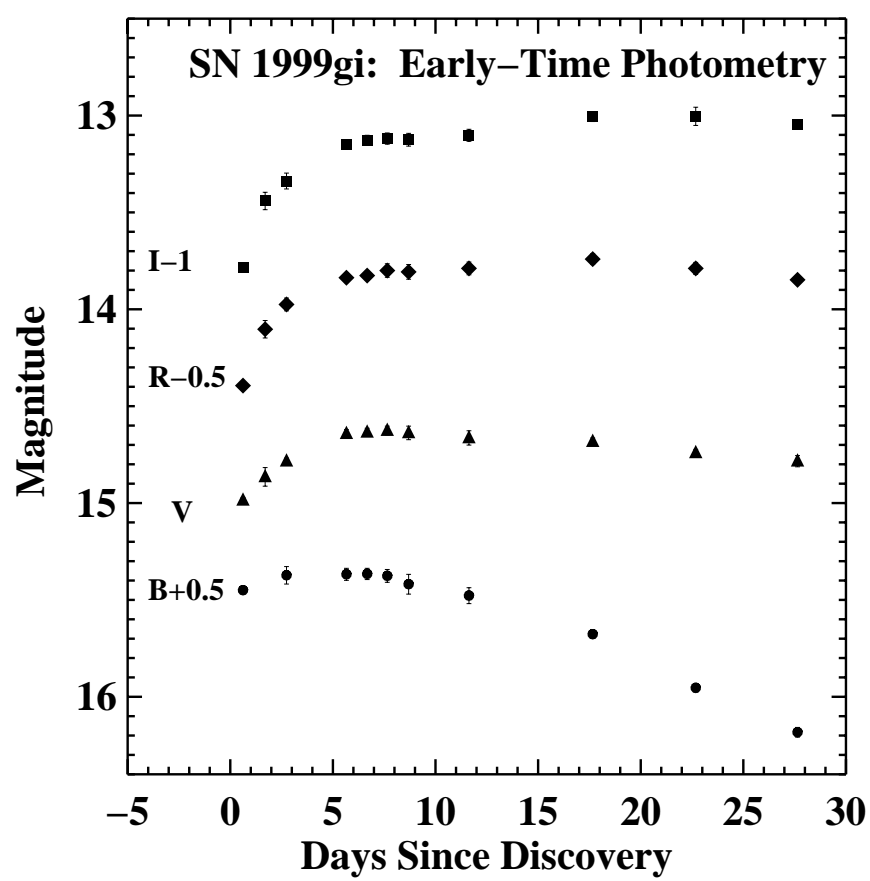

FIG. 3.-Detail of the $B V R I$ light curves presented in Fig. 2 showing the first 30 days of photometric development for SN 1999gi. 
$m_{V}(\max ) \approx 14.6 \mathrm{mag}$ on HJD $2,451,530.0 \pm 1.6$ (1999 December $17.5 \pm 1.6$ ), which is $7.7 \pm 1.6$ days after discovery. The decline in the $B$ band over the first 100 days after maximum light is $\beta_{100}^{B}=1.9 \mathrm{mag}$, which definitively establishes SN 1999gi as a Type II-P event according to the definition by Patat et al. (1994) used to discriminate $\mathrm{SNe}$ II-P $\left(\beta_{100}^{B}<3.5 \mathrm{mag}\right)$ from SNe II-L $\left(\beta_{100}^{B}>3.5 \mathrm{mag}\right)$. The average apparent $V$-band brightness during the plateau, defined as the unweighted mean of the values from days 20 to 100 after the explosion (determined by the EPM analysis in $\S 3)$, is $\langle m\rangle_{V}$ (plateau) $=14.90$ mag.

The photometric behavior of SN 1999gi is extremely similar to that of SN $1999 \mathrm{em}$, with both having plateau durations of $\sim 95$ days after $B$-band maximum, as well as similar $B$-band decline rates (see L02). In fact, once an allowance is made for a small reddening difference $\left(\Delta E(B-V) \equiv\left[E(B-V)_{99 \mathrm{gi}}-E(B-V)_{99 \mathrm{em}}\right]=0.15 \mathrm{mag}\right)$, the color evolution of the two objects is nearly identical (Fig. 4). The optical "double peak" noted by L02 for SN $1999 \mathrm{em}$, in which a second local maximum follows the initial peak $(8.6 \pm 2.3$ days later for the $V$-band in $\mathrm{SN}$ $1999 \mathrm{em}$ ), is also discernible in the photometry of SN 1999gi (see Fig. 3). However, the second peak is certainly not as obvious as it was in the photometry of SN $1999 \mathrm{em}$, a situation likely exacerbated by the relatively sparse photometric coverage obtained during this period for SN 1999gi compared with SN 1999em.

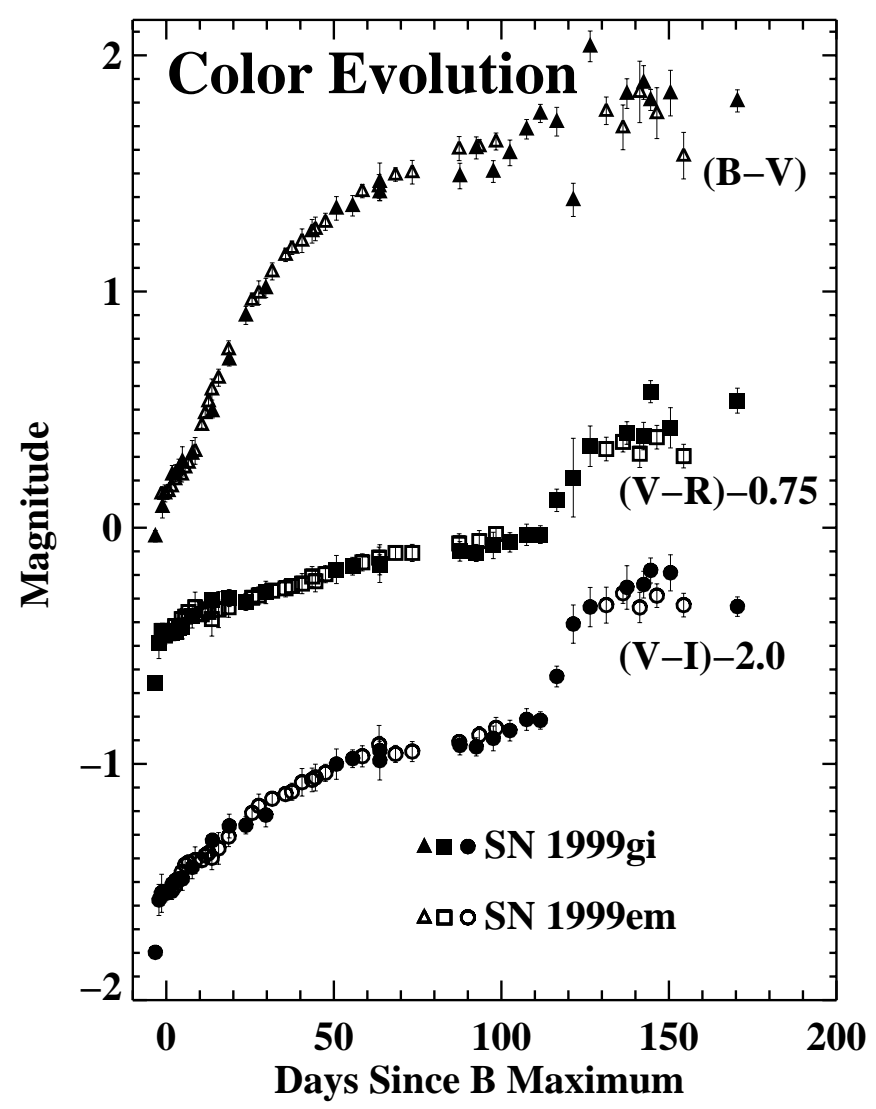

FIG. 4. $-B-V, V-R$, and $V-I$ color curves of SN 1999gi ( filled symbols) compared with SN 1999em (open symbols; from L02), plotted against the time since $B$-band maximum light (3.9 and 2.4 days after discovery for SN 1999gi and SN 1999em, respectively). The data for SN 1999em have been artificially reddened by an additional $E(B-V)=0.15 \mathrm{mag}$.

\subsection{Spectroscopy}

Table 3 lists the spectral observations of SN 1999gi. All one-dimensional sky-subtracted spectra were extracted optimally (Horne 1986) in the usual manner. Each spectrum was then wavelength and flux calibrated, as well as corrected for continuum atmospheric extinction and telluric absorption bands (Wade \& Horne 1988; Bessell 1999; Matheson et al. 2000). With the exception of the very first spectrum (day $0.61,300 \mathrm{~s}$ observation), all spectra were observed near the parallactic angle (Filippenko 1982), so the spectral shape should be quite accurate. ${ }^{9}$

The spectral evolution of SN 1999gi during the first 168 days of its development is shown in Figure 5, and it is very similar to that seen for SN 1999em (L02; see also Leonard \& Filippenko 2001, Fig. 10). As is typical for SNe II-P, the early-time spectrum is characterized by a smooth thermal continuum with superposed hydrogen Balmer and $\mathrm{He}$ I $\lambda 5876$ P Cygni lines. During the plateau phase numerous metal-line P Cygni features complicate the spectrum (see L02 for a complete identification of these features). Emission-dominated lines then become prominent as SN 1999gi drops off the plateau and makes the transition to the nebular phase.

To estimate the recession velocity of SN 1999gi, we measured the peak wavelength of the narrow $\mathrm{H} \alpha$ line resulting from the superposed $\mathrm{H}$ II region. From this we determine that $v_{\mathrm{rec}}=552 \pm 10 \mathrm{~km} \mathrm{~s}^{-1}$, where the uncertainty is the $1 \sigma$

${ }^{9}$ A direct comparison of the first spectrum with the one taken immediately after it at the parallactic angle (day $0.62,600 \mathrm{~s}$ observation) confirms that the low air mass (1.14) of this observation renders the effects of differential light loss negligible.

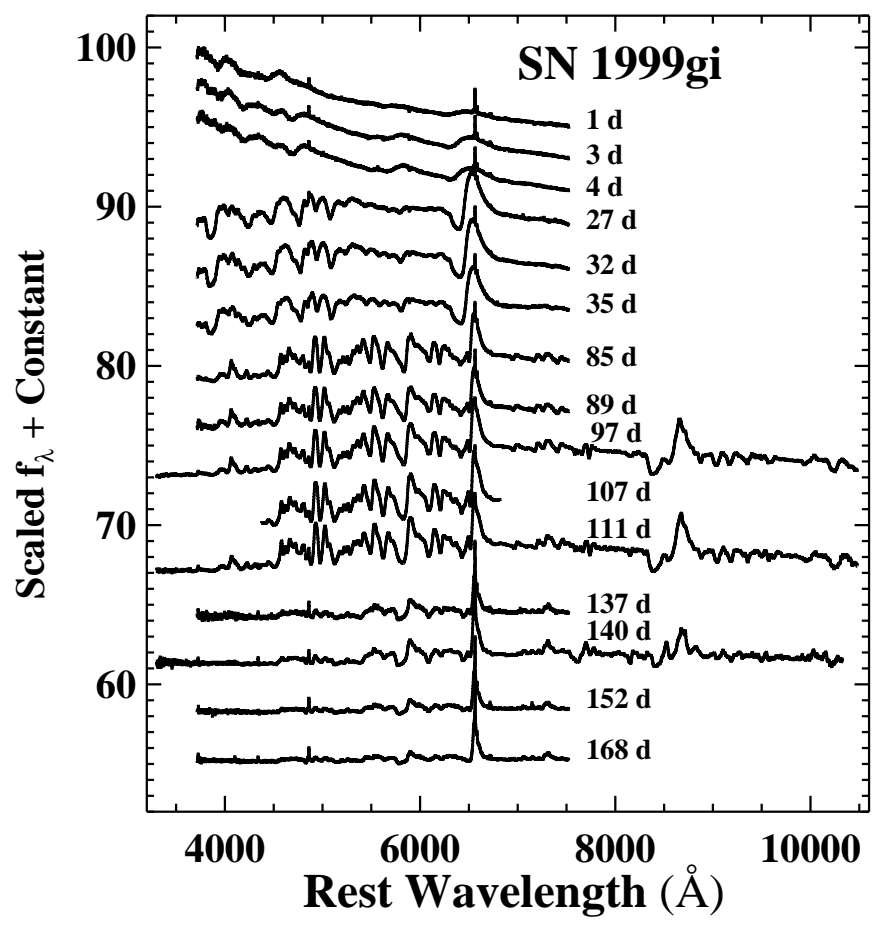

FIG. 5.-Optical flux spectra of SN 1999gi with day since discovery indicated. Note the narrow emission spike evident near the peaks of the $\mathrm{H} \alpha$ $(6563 \AA$ ) and $H \beta$ (4861 $\AA$ ) profiles due to a superposed H II region. In this and all figures a recession velocity of $552 \mathrm{~km} \mathrm{~s}^{-1}$ has been removed from the observed spectrum $(\S 2.2)$. 


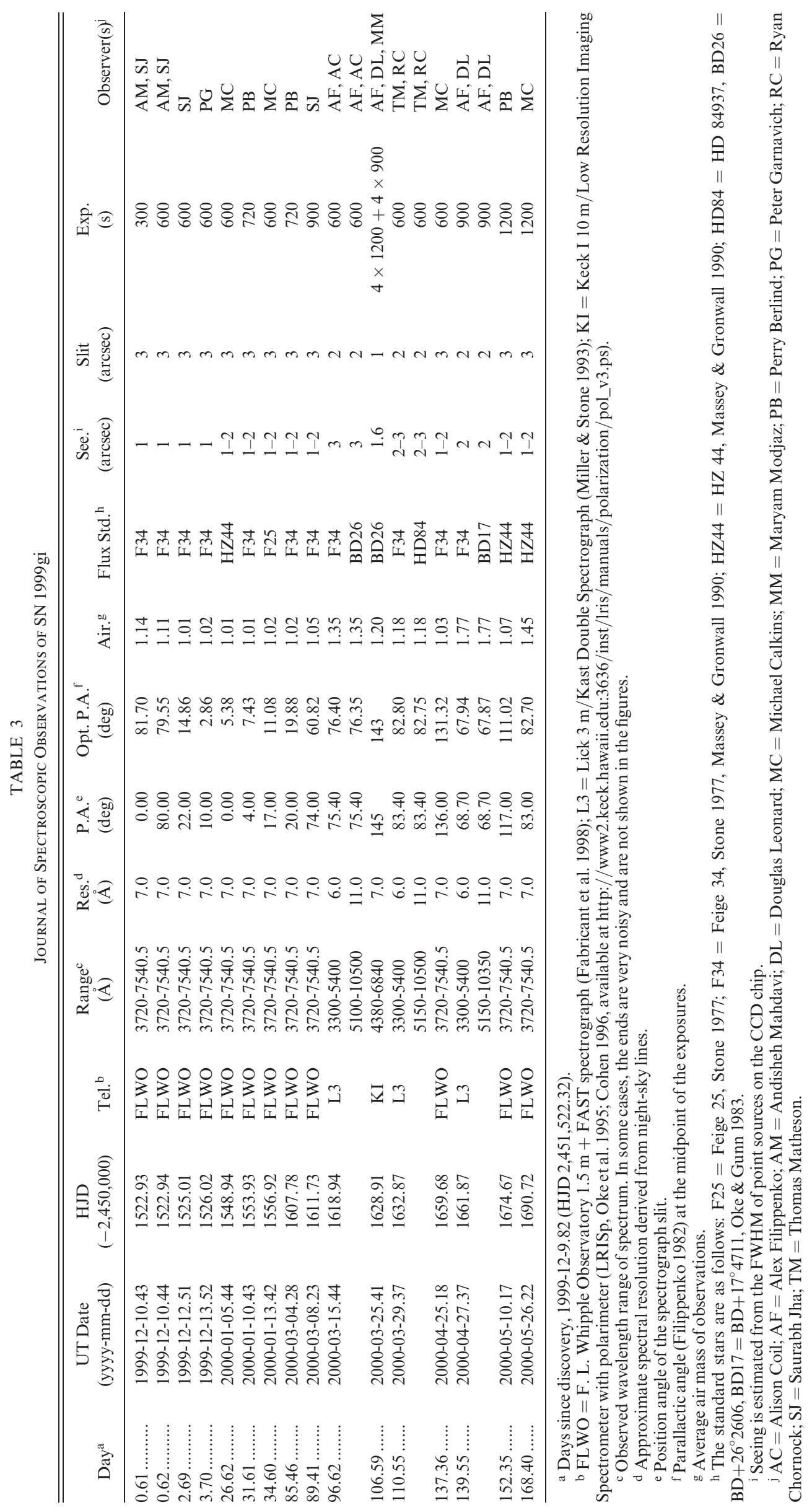


spread in the velocities derived from the individual spectra. This velocity is to be compared with the value reported for NGC 3184 by Strauss et al. (1992) of $592 \pm 1 \mathrm{~km} \mathrm{~s}^{-1}$, which was derived from narrow $\mathrm{H}$ II emission in the central $10^{\prime \prime}$ of the galaxy. Since SN 1999gi is quite far away from the center of NGC 3184 (Fig. 1), our velocity is likely a more accurate estimate of the true velocity of the $\mathrm{SN}$. We therefore use it to derive all photospheric velocities in the EPM analysis in $\S 3$; ultimately, using the larger Strauss et al. (1992) value would result in a final EPM distance that is $\sim 1 \%$ larger than that derived with our lower recession velocity.

The very early time $\mathrm{H} \beta$ and $\mathrm{He}$ I $\lambda 5876$ line profiles of $\mathrm{SN}$ 1999gi are shown in Figure 6, and they warrant special comment. In early-time spectra of SN $1999 \mathrm{em}$ Baron et al. (2000) identify "complicated P Cygni" profiles, in which the usual $\mathrm{P}$ Cygni absorptions for $\mathrm{H} \beta$ and $\mathrm{He}$ I $\lambda 5876$ are accompanied by a second absorption at much higher velocity. From comparison with theoretical models Baron et al. (2000) conclude that these high-velocity absorption features result from nonlocal thermodynamic equilibrium effects that produce two line-forming regions in the expanding

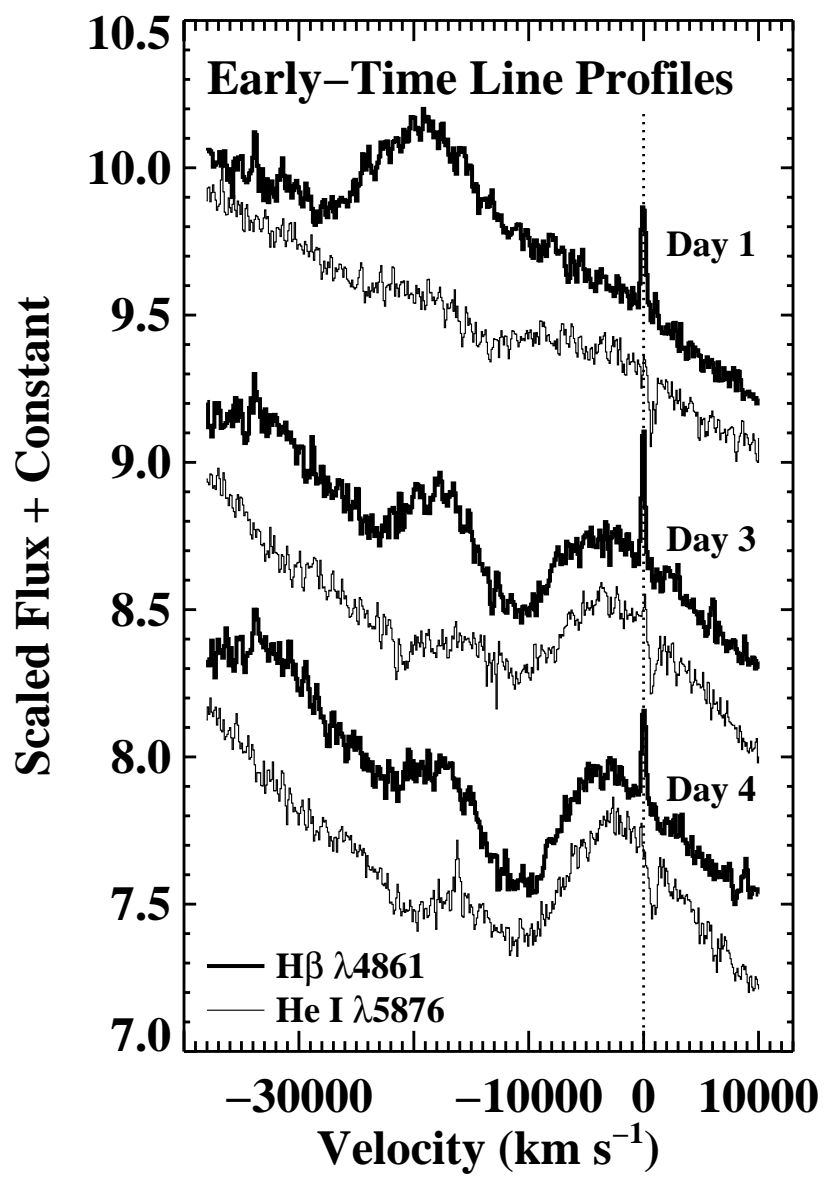

FIG. 6.-Early-time development of the $\mathrm{H} \beta$ (thick lines) and $\mathrm{He}$ I $\lambda 5876$ (thin lines) profiles, with days since discovery indicated. Note the highvelocity $\left(v>20,000 \mathrm{~km} \mathrm{~s}^{-1}\right) \mathrm{P}$ Cygni absorption that exists in addition to the "normal," lower velocity $\left(v \approx 10,000 \mathrm{~km} \mathrm{~s}^{-1}\right)$ profiles in both lines. In the first spectrum, in fact, the high-velocity component of $\mathrm{H} \beta$ dominates over the weaker, low-velocity profile. Note also the narrow emission spike at zero velocity in the $\mathrm{H} \beta$ profiles due to a superposed $\mathrm{H}$ II region, the sharp, narrow absorption feature just redward of zero velocity in the $\mathrm{He}$ I $\lambda 5876$ spectra from Na I D gas in NGC 3184, and the small spike at $v \approx-16,000$ $\mathrm{km} \mathrm{s}^{-1}$ in the day $4 \mathrm{He}$ I $\lambda 5876$ profile from poor telluric [O $\left.\mathrm{I}\right] \lambda 5577$ removal. atmosphere. Leonard et al. (2001) also identify these highvelocity features in an early spectrum of SN $1999 \mathrm{em}$. In addition, L02 propose that complex P Cygni profiles of strong lines may also explain a number of previously unidentified absorption features in spectra of SN 1999em at later times, during the photospheric and early nebular phases; detailed modeling, however, is required to confirm these identifications. The early-time absorption features observed in the spectra of SN 1999em were rather subtle and left open the question of whether they were true P Cygni features containing both a dip and a peak. As clearly seen in Figure 6, similar high-velocity features are also evident in the early spectra of SN 1999gi. Furthermore, the $\mathrm{H} \beta$ profiles from days 3 and 4 reveal that the high-velocity feature is indeed characterized by a true P Cygni profile.

The earliest spectrum, from day 1 , is particularly interesting. In $\mathrm{He}$ I $\lambda 5876$ there is just a hint of the higher velocity component in addition to the "normal" line feature. Near the expected location of the high-velocity feature in $\mathrm{H} \beta$ (i.e., based on its position in the spectra from days 3 and 4 ), there is a strong emission feature, which, if identified with $\mathrm{H} \beta$, extends out to nearly $-30,000 \mathrm{~km} \mathrm{~s}^{-1}$. If this association is confirmed by detailed models, it would demonstrate the existence of very high velocity material in the outer envelope of SN 1999gi at these early times. Although the "normal," lower velocity component of $\mathrm{H} \beta$ is likely present at this early epoch, it is clearly much weaker than the higher velocity component. ${ }^{10}$ Interestingly, near the expected location of $\mathrm{H} \alpha$ we find that, while the "normal" profile is observed at all epochs (including day 1), a high-velocity feature is not apparent in the spectra from days 1,3, and 4. At later times, during the photospheric and early nebular phases, the highvelocity absorption features attributed by $\mathrm{L} 02$ to $\mathrm{H} \beta$, Na I $\mathrm{D}$, and $\mathrm{H} \alpha$ in spectra of SN $1999 \mathrm{em}$ are also seen in the spectra of SN 1999gi: They are consistently detected in $\mathrm{H} \beta$ and $\mathrm{Na}$ I $\mathrm{D}$ at $9000 \lesssim v \lesssim 11,000 \mathrm{~km} \mathrm{~s}^{-1}$ through the spectrum on day 111, and are obvious in $\mathrm{H} \alpha$ (at $v \approx 13,700 \mathrm{~km} \mathrm{~s}^{-1}$ ) in the spectra on days 32 and 35. Further detailed modeling is urged in order to better establish the physical mechanism behind these intriguing features.

\subsection{The Reddening of SN $1999 \mathrm{gi}$}

SN 1999gi has minimal Galactic reddening, $E(B-V)=$ 0.017 mag (Schlegel, Finkbeiner, \& Davis 1998). In order to estimate the total reddening (or to set limits on how high it can be), we shall compare the results obtained from the following five independent techniques.

1. Compare the early-time spectral shape with blackbody functions. As discussed by Eastman, Schmidt, \& Kirshner (1996), an upper reddening limit can be set on an SN II-P by comparing its very early time spectral shape with an arbitrarily hot blackbody function. The basic idea is that, at very high temperatures (i.e., early times for an SN II-P), the optical continuum is on the Rayleigh-Jeans tail of the Planck spectrum, whose slope is quite insensitive to temperature (i.e., in the limit $T \rightarrow \infty, \partial \ln f_{\lambda} / \partial \ln \lambda \approx-4$ ). If a hot, early-time spectrum (with accurate continuum shape) is

${ }^{10}$ Although not shown in the figure, we note that there is also a strong feature at the expected location of a high-velocity component for $\mathrm{H} \gamma$, but the identification is ambiguous, since the lower velocity absorption of $\mathrm{H} \delta$ is expected at nearly the same location. 
available (the earlier, the better, since cooler spectra will result in a less restrictive upper bound), one can determine the maximum allowable reddening by dereddening the spectrum to the point at which even an unphysically hot blackbody function (say, $10^{9} \mathrm{~K}$ ) can no longer fit the continuum. As shown in Figure 7, the inability of a blackbody at any temperature to match the continuum shape of SN 1999gi when dereddened by $E(B-V)>0.45$ mag effectively establishes this as an upper limit for the reddening of SN 1999gi. The true reddening of the $\mathrm{SN}$, of course, is likely to be substantially lower. For instance, the more reasonable color temperature of 19,500 K is shown in Figure 7 to correspond with a reddening of $E(B-V)=0.21 \mathrm{mag}$.

2. Compare the color evolution of $S N 1999 \mathrm{gi}$ with $S N$ $1999 \mathrm{em}$. Schmidt, Kirshner, \& Eastman (1992) demonstrate that the color evolution of the five SNe II-P in their study is

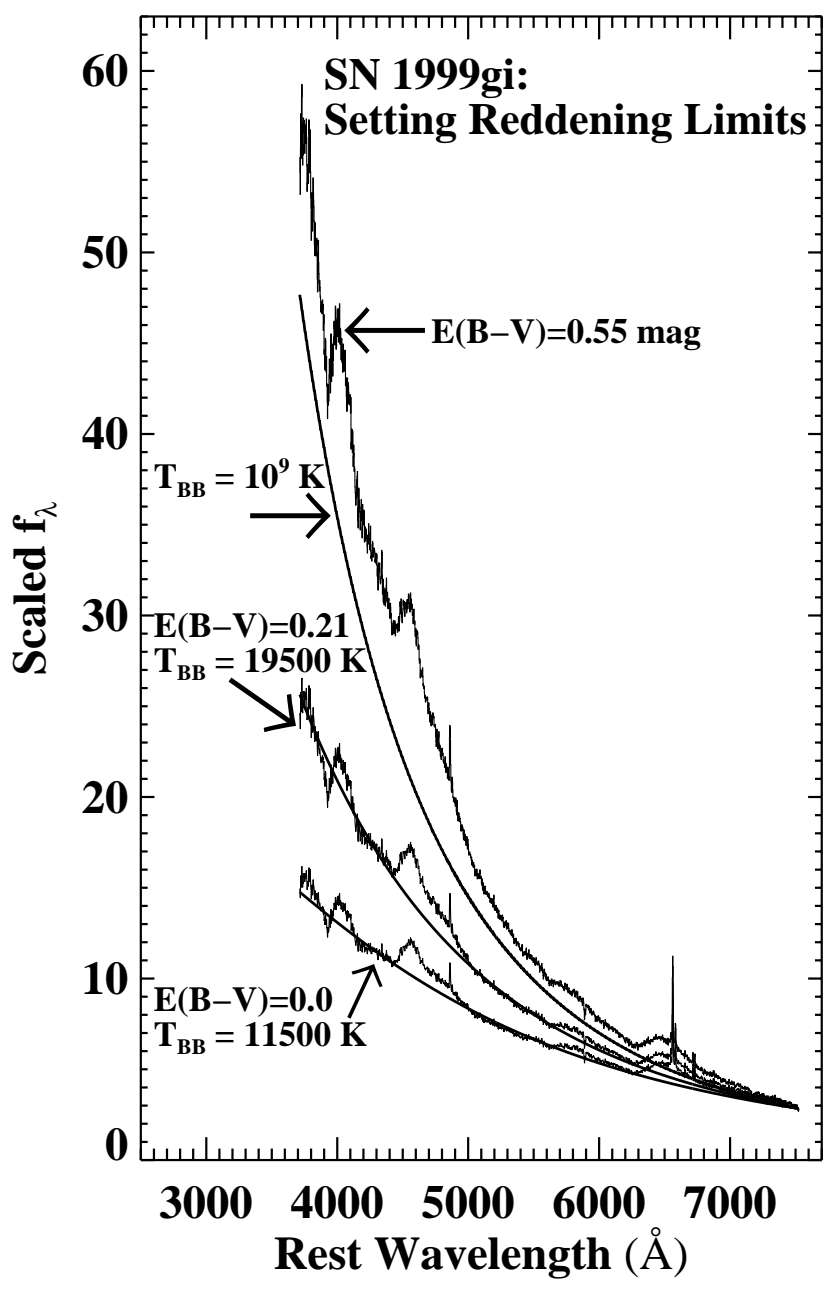

FIG. 7.-Flux spectrum of SN 1999gi taken 0.62 days after discovery, dereddened by $E(B-V)=0.55,0.21$, and $0.00 \mathrm{mag}$ (top, middle, and bottom, respectively), with blackbody functions at the indicated temperatures also shown. For assumed reddening values of $E(B-V)<0.45 \mathrm{mag}$, it is possible to trade increases in reddening with increases in temperature. However, when the spectrum is dereddened by $E(B-V)>0.45 \mathrm{mag}$ [represented here by $E(B-V)=0.55 \mathrm{mag}$ ], the spectral slope of SN 1999gi cannot be matched by a blackbody at any temperature, no matter how hot (represented here by $10^{9} \mathrm{~K}$ ). The inability of an arbitrarily hot blackbody to match the continuum slope for $E(B-V)>0.45$ mag effectively establishes $0.45 \mathrm{mag}$ as an upper reddening limit. For ease of comparison, the lower two spectra have been scaled to match the flux level of the top spectrum at the red end. quite similar, with the observed discrepancies consistent with reddening differences among the objects. Although still limited by the small number of well-studied examples, it is tempting to posit that all SNe II-P undergo similar color evolution, at least through the end of the recombination phase. If this is so, then it should be possible to estimate (and set hard upper limits on) the reddening of SN 1999gi by comparing it with SN 1999em, which was extensively modeled by Baron et al. (2000), who set a firm upper limit of $E(B-V)_{\text {tot }}<0.15 \mathrm{mag}$ and estimated the likely reddening to be $0.05 \lesssim E(B-V)_{\text {tot }} \lesssim 0.10$ mag. (The EPM analyses of Hamuy et al. 2001 and L02 are also consistent with low reddening for SN 1999em.) The similar color evolution shown in Figure 4, where the data for both objects are plotted with respect to the date of $B$-band maximum, suggests a reddening difference between the two objects of $\triangle E(B-V)=0.15$ mag, implying that $0.20 \lesssim E(B-V)_{99 \mathrm{gi}} \lesssim 0.25 \mathrm{mag}$, with $E(B-V)_{99 \mathrm{gi}}<0.30 \mathrm{mag}$ serving as a hard upper limit. Of course, this analysis assumes the intrinsic color evolution of the two objects to be identical with respect to the date of maximum brightness. If the analysis is instead done with respect to the explosion date (derived from the EPM analyses), then the best agreement is found for $0.15 \lesssim E(B-V)_{99 \mathrm{gi}} \lesssim 0.20 \mathrm{mag}$, in which case $E(B-V)<$ 0.25 mag then becomes the upper bound. We therefore conclude that the comparison of the color evolution of $\mathrm{SN}$ 1999 gi with that of SN $1999 \mathrm{em}$ implies a reddening of $E(B-V) \approx 0.20 \mathrm{mag}$ for SN 1999gi.

3. Translate observed $\mathrm{Na}$ I $\mathrm{D}$ interstellar absorption equivalent width into a reddening estimate. The correlations between the $\mathrm{Na}$ I $\mathrm{D} \lambda \lambda 5890,5896$ interstellar line equivalent width and reddening found by Barbon et al. (1990) and Munari \& Zwitter (1997) are known to have a very large scatter, especially when only low-resolution spectra are available (see Leonard \& Filippenko 2001 for a more extensive discussion of the utility of these relations). Nevertheless, it is useful to compare the predictions that these relations make with the results of the other, perhaps more accurate, techniques.

To derive the $\mathrm{Na}$ I $\mathrm{D}$ equivalent width in the spectrum of SN 1999gi, we first co-added the normalized region around the $\mathrm{Na}$ I $\mathrm{D}$ lines in the five earliest spectra, which had the highest signal-to-noise ratio $(\mathrm{S} / \mathrm{N})$ in the region of interest (i.e., we combined the spectra up through and including the one taken on day 31.61; the spectrum from day 34.60 was corrupted by a cosmic-ray spike in the $\mathrm{Na}$ I $\mathrm{D}$ region, and the high-S/N spectrum from day 106.59 could not be used with confidence since the $\mathrm{Na}$ I $\mathrm{D}$ absorption occurred very near the maximum of an SN feature, making proper continuum placement difficult). From this composite spectrum we measure $W_{\lambda}(\mathrm{Na}$ I $\mathrm{D})=0.83 \pm 0.15 \AA$, with $W_{\lambda}(\mathrm{Na}$ I $\mathrm{D} 1[\lambda 5896])=0.31 \pm 0.15 \AA$, and $W_{\lambda}(\mathrm{Na}$ I $\mathrm{D} 2$ $[\lambda 5890])=0.47 \pm 0.20 \AA$; the uncertainty in the measurements represents the $1 \sigma$ spread in the automated measurements of 1000 sets of simulated data characterized by the $\mathrm{S} / \mathrm{N}$ of the composite spectrum. To solve for the D1 and D2 components separately, we used the Levenberg-Marquardt maximization algorithm (Press et al. 1992) with Gaussians constrained to have identical widths (set equal to the spectral resolution) and separated by $5.97 \mathrm{~A}$. Note that, since the $W_{\lambda}(\mathrm{Na}$ I $\mathrm{D} 1[\lambda 5896])$ and $W_{\lambda}(\mathrm{Na}$ I $\mathrm{D} 2[\lambda 5890])$ values are the result of model fits, whereas $W_{\lambda}(\mathrm{Na}$ I D) is measured directly from the (noisy) spectrum, the sum of the equivalent widths of the individual lines does not exactly equal the 
reported total equivalent width (but is equal within the uncertainty). From these values, the Barbon (1990) relation yields $E(B-V)_{\text {host }} \approx 0.21 \mathrm{mag}$, and the Munari \& Zwitter (1997) relation gives $E(B-V)_{\text {host }} \approx 0.27 \mathrm{mag}$. From a similarly constructed composite spectrum centered on the expected location of the Na I D lines in the Milky Way (i.e., at zero velocity), the absence of detectable absorption implies an upper limit on the total equivalent width due to Galactic gas of $W_{\lambda}(3 \sigma)=0.06 \AA$ through equation (4) of Leonard \& Filippenko (2001); this translates into an upper reddening limit of $E(B-V)_{\mathrm{MW}}<0.02 \mathrm{mag}$ from the Barbon (1990) relation, which concurs with the value given by Schlegel et al. (1998). The total reddening predicted by these crude relations is therefore found to be $E(B-V) \approx 0.25$ mag, a value that is consistent with the upper limits and values derived by the other methods.

4. Assume the reddening to SN 1999gi to be the same as that derived to the young $O B$ association in which it is situated. From analysis of WFPC2 HST observations of NGC 3184, S01 conclude that SN 1999gi originates in a resolved, young $\mathrm{OB}$ association. By comparing the evolutionary isochrones of Lejuene \& Schaerer (2001) with the colormagnitude diagram of the stars in the $\mathrm{OB}$ association (i.e., those within 12 ".5 of SN 1999gi), S01 find $E(B-V)=0.15$ mag to give the best fit to the cluster and note that for reddenings above $E(B-V)=0.30 \mathrm{mag}$ the isochrones fail to match the color of the brightest sources.

5. Assume the reddening to SN 1999gi to be the same as that derived to a nearby $H$ II region. In order to estimate nebular abundances in NGC 3184, Zaritsky, Kennicutt, \& Huchra (1994) determined the reddening toward $19 \mathrm{H}$ II regions, one of which is just 8.5 away from SN 1999gi and located in the OB association identified by S01 to likely contain the progenitor of SN 1999gi. Assuming $R_{V}=3.1$ (Savage \& Mathis 1979), Zaritsky et al. (1994) find $E(B-V)=0.34$ mag to this nearby $\mathrm{H}$ II region.

None of the techniques that we have used to estimate the total reddening to SN 1999gi is individually unassailable. Potential weaknesses of each technique are as follows (given for each of the techniques, respectively): (1) Early-time SN II-P color may depart slightly from a single-temperature blackbody. (2) All SNe II-P may not evolve in a similar manner, and SN 1999gi and SN 1999em may not have been identical events. (3) Sodium is known to be only a fair tracer of dust, and our low-resolution spectra do not resolve the individual absorption systems that contribute to the $\mathrm{Na}$ I D lines, making it impossible to estimate the effect of line saturation. (4) and (5) The reddening along the specific line of sight to SN 1999gi may differ from the average reddening to the $\mathrm{OB}$ association and/or the reddening to specific association members (see, e.g., Yadav \& Sagar 2001). Nonetheless, taken together the general agreement of all the estimates makes a compelling case for low reddening to SN 1999gi, with $E(B-V)=0.20 \mathrm{mag}$ (comparison with SN 1999em); $0.25 \mathrm{mag}$ ( $\mathrm{Na}$ I D absorption); $0.15 \mathrm{mag}$ (reddening to $\mathrm{OB}$ association); and 0.34 (reddening to $\mathrm{H}$ II region), and an upper reddening limit of $E(B-V)<0.45$ mag coming from the analysis of the early-time continuum shape. If the color evolution of SN 1999gi is intrinsically the same as that of SN 1999em with respect to the time of maximum light, then we can restrict the upper bound even further, to $E(B-V)<0.30 \mathrm{mag}$. In $\S 3$ we shall see that additional evidence for low reddening to SN 1999gi comes from the EPM analysis itself, which predicts $E(B-V) \approx 0.10$ mag. Taking the simple average of the five specific reddening estimates (i.e., including the EPM estimate derived in $\S 3$ but not incorporating the upper limit derived from the blackbody comparison) yields $E(B-V)=0.21 \pm 0.09 \mathrm{mag}$, where the uncertainty is the $1 \sigma$ spread of the individual reddening estimates from the mean. We adopt this value as our best estimate of the reddening of SN 1999gi.

\section{THE EPM APPLIED TO SN 1999gi}

To derive the EPM distance to SN 1999gi we follow the procedure detailed by L02. Briefly, an EPM distance is calculated by comparing the linear radius of the expanding supernova photosphere, $R$, with the photosphere's angular size, $\theta$, to derive the distance to the $\mathrm{SN}, D$ (Kirshner \& Kwan 1974). The radial velocity of the expanding photosphere, $v$, is found from the Doppler shifting of the spectral lines, so that $R=v\left(t-t_{0}\right)$, where $\left(t-t_{0}\right)$ is the time since explosion and the $\mathrm{SN}$ is assumed to be in free expansion. The photosphere's theoretical angular size, $\theta$, is calculated by comparing the observed flux with that predicted from theoretical models (i.e., a "dilute" blackbody; Eastman et al. 1996) for a spherical SN photosphere, as

$$
\theta=\sqrt{\frac{f_{\nu} 10^{0.4 A_{\nu}}}{\zeta_{\nu}^{2}\left(T_{c}\right) \pi B_{\nu}\left(T_{c}\right)}},
$$

where $B$ is the Planck function at color temperature $T_{c}, f_{\nu}$ is the flux density received at Earth, $A$ is the extinction, and $\zeta\left(T_{c}\right)$ is the color temperature dependent "dilution factor" (or "distance correction factor," since it " corrects" derived distances such that $D_{\text {actual }}=\zeta D_{\text {measured; }}$ see L02 for a thorough discussion of this term). Since accurate spectrophotometry is generally not available, equation (2) is typically recast in terms of broadband photometry, with $T_{c}$ and $\zeta$ derived for some subset of $B V I J H K$. With $R$ and $\theta$ known, $D$ can be found, since $\theta=R / D$ in the small-angle approximation. Substituting $v\left(t-t_{0}\right)$ for $R$ and rearranging, we arrive at

$$
t=D\left(\frac{\theta}{v_{\text {phot }}}\right)+t_{0} .
$$

A plot of $\theta / v_{\text {phot }}$ against $t$ should therefore result in a line with slope $D$ and $y$-intercept $t_{0}$.

As discussed by L02, the overall level of the theoretically derived dilution factor $\zeta\left(T_{c}\right)$ has long been touted as potentially the largest source of systematic uncertainty in the application of the EPM to SNe II-P, due mainly to the fact that most applications of the EPM have relied on the dilution factors produced by only one modeling group (i.e., Eastman et al. 1996). Since no other independent modeling group has published dilution factors appropriate for SNe II-P, ${ }^{11}$ it is difficult to quantify the amount of systematic uncertainty that the reliance on only one group's dilution

\footnotetext{
${ }^{11}$ Eastman et al. (1996) stress that their dilution factors are only appropriate for SNe II-P and not to peculiar variants of the SN II subclass. Eastman et al. (1996) also note that the models of Montes \& Wagoner (1995), while appropriate for SNe II-P, are difficult to directly compare with their results, since Montes \& Wagoner (1995) define $\zeta$ somewhat differently and also do not model the effect that line blanketing has on the apparent color temperature.
} 
factors contributes to EPM distances. However, to get a sense of the possible differences among modeling groups, it is instructive to consider the two unusual (i.e., not SNe II-P) core-collapse events for which independent dilution factors from another group have been derived: SN 1987A and SN 1993J.

Recently, Mitchell et al. (2002) derived a distance to SN 1987A of $D=50 \pm 5 \mathrm{kpc}$ through detailed modeling of the spectra and the use of the spectral-fitting expanding atmosphere method (SEAM; Baron et al. 1993; Baron, Hauschildt, \& Branch 1994; Baron et al. 1995). This distance is in excellent agreement with other, independent distance estimates to SN 1987A and the Large Magellanic Cloud (see, e.g., Gibson 2000). SN 1987A was not a classical SN II-P: its progenitor was a compact blue supergiant, and its light curve became powered by radioactivity substantially earlier than is typical for SNe II-P, which result from progenitors with more extended envelopes. Nonetheless, Mitchell et al. (2002) do provide a direct comparison between the dilution factors resulting from their SN 1987Alike models with two of the SN II-P models by Eastman et al. (1996) for dilution factors derived using the $B V$ bandpasses to estimate the color temperature. While they find quite good agreement between the dilution factors at both high $(T \gtrsim 8000 \mathrm{~K})$ and low $(T \lesssim 5000 \mathrm{~K})$ temperatures, they note some disagreement in the important temperature range of $5000-8000 \mathrm{~K}$. In this region the dilution factors of Mitchell et al. (2002) are $\sim 50 \%-60 \%$ larger than those of Eastman et al. (1996). This implies that using the dilution factors of Eastman et al. (1996), then, would result in a shorter distance to SN 1987 A (e.g., 50\%-60\% smaller if only this temperature range were considered) than that found by using the Mitchell et al. (2002) models. Recently, Hamuy (2001) has derived the EPM distance to SN 1987A using the dilution factors of Eastman et al. (1996), as slightly modified for the effects of telluric absorption by Hamuy et al. (2001). As anticipated from the comparison by Mitchell et al. (2002), Hamuy (2001) indeed finds a shorter distance to SN 1987 A. Using the dilution factors for the $B V$ filter combination (while noting that these dilution factors are not entirely appropriate for SN 1987A), Hamuy (2001) finds $D=37$ kpc. The discrepancy between the two results may be due to the unusual nature of SN 1987A and not to fundamental differences in the modeling results. In further support of this notion, we note that, when Eastman \& Kirshner (1989) derived an EPM distance to SN 1987A with models custom crafted for this unusual event, they found $D=49 \pm 6 \mathrm{kpc}$, a value that is quite consistent with the Mitchell et al. (2002) value.

In a similar manner, Baron et al. (1995) ${ }^{12}$ derive a SEAM distance to SN 1993J, a "Type IIb" event (Filippenko 1988) thought to arise from a progenitor that exploded with just a low-mass outer layer of hydrogen remaining, since the spectrum showed hydrogen at early times but subsequently became helium dominated (see Matheson et al. 2000 and references therein). Using models customized to match the observed spectra of SN 1993J, Baron et al. (1995) derive a distance of $4.0 \pm 0.6 \mathrm{Mpc}$, which is in good agreement with the Cepheid distance (corrected for metallicity effects)

\footnotetext{
12 Note that Baron et al. (1995) and Mitchell et al. (2002) both employ the same radiative transfer code, PHOENIX (Baron et al. 1994), whereas the Eastman et al. (1996) values were derived using the radiative transport code EDDINGTON (Eastman \& Pinto 1993).
}

recently reported by Freedman et al. (2001) to its host, M81, of $3.63 \pm 0.13 \mathrm{Mpc}$. Baron et al. (1995) compare the dilution factors derived from their models of SN 1993J with those of Schmidt (1993), in which a preliminary version of the dilution factors ultimately published by Eastman et al. (1996) was presented. Although it was not possible to make a detailed comparison at the time (Schmidt only presents the dilution factors in graphical form), Baron et al. (1995) noted that their $\zeta\left(T_{c}\right)$ values for SN 1993J were generally greater than those of Schmidt (1993) by about 60\%. This implies that applying the EPM to SN 1993J with the dilution factors appropriate for an SN II-P should lead to an underestimate of the distance. Indeed, when Schmidt et al. (1993) apply the dilution factors of Schmidt (1993) to SN $1993 \mathrm{~J}$, they find $D=2.6 \pm 0.4 \mathrm{Mpc}$, a value $\sim 50 \%$ closer than that derived by Baron et al. (1995). As was the case with Hamuy (2001) for SN 1987A, Schmidt et al. (1993) point out the potential danger in applying the dilution factors crafted for SNe II-P to SN 1993J, concluding that "supernova models that more closely match the atypical spectral features of SN 1993J may change the inferred distance."

Therefore, although discrepancies exist between the dilution factors presented by Eastman et al. (1996) and those provided by one other group for SN 1987A and SN 1993J, we conclude that at least some of the observed difference may be explained by the fact that Eastman et al. (1996) specifically modeled SNe II-P atmospheres, whereas the SNe modeled by the other group were both peculiar SN II events. Lacking a direct comparison between other modeling groups' values for the dilution factors derived specifically to SNe II-P and those presented by Eastman et al. (1996), it remains difficult to quantify the degree of systematic uncertainty that the dilution factor adds to distances determined to SNe II-P at this time; a direct comparison of the dilution factors between modeling groups for SN II-P atmospheres is urged to help better quantify this potential source of systematic uncertainty in the EPM technique.

For our study of SN 1999gi, by all accounts a "normal" SN II-P, we shall use the dilution factors of Eastman et al. (1996) as slightly modified by Hamuy et al. (2001), for the $B V, B V I$, and $V I$ filter combinations. As recommended by L02, we determine the photospheric velocity of SN 1999gi at each spectral epoch by taking the weighted average of the blueshift of the absorption troughs of the available weak, unblended line features due to Fe II $\lambda 4629$, Sc II $\lambda 4670, \mathrm{Fe}$ II $\lambda 5276$, and $\mathrm{Fe}$ II $\lambda 5318$. These features are not present in the three earliest spectra, so we follow L02 and use the weakest available lines that are found to yield the lowest consistent velocity at these times, namely $\mathrm{H} \delta, \mathrm{H} \gamma, \mathrm{H} \beta$, and $\mathrm{He}$ I $\lambda 5876$. The BVI magnitudes of SN 1999gi at each of the spectral epochs were derived through interpolation from the nearby values. Table 4 lists the values of the parameters used in the EPM analysis.

Because reddening affects the inferred color temperature as well as the apparent SN brightness, the value of $\theta$ (eq. [2]) and, therefore, the derived distance and time of explosion will surely have a reddening dependence. One strength of the EPM technique is that the derived distance should be rather robust to uncertainty in reddening, since both $f$ and $B$ in equation (2) have similar dependencies: higher reddening decreases both the observed flux and the color temperature in a smooth, regular manner. However, one must also consider the temperature dependence of the dilution factor $\zeta$, 
TABLE 4

QuANTITIES UsEd IN THE EPM ANALYSIS OF SN 1999gi

\begin{tabular}{|c|c|c|c|c|}
\hline Day $^{\mathrm{a}}$ & $\begin{array}{c}v\left(\sigma_{v}\right) \\
\left(\mathrm{km} \mathrm{s}^{-1}\right)\end{array}$ & $\begin{array}{l}B\left(\sigma_{B}\right) \\
(\mathrm{mag})\end{array}$ & $\begin{array}{l}V\left(\sigma_{V}\right) \\
(\mathrm{mag})\end{array}$ & $\begin{array}{l}I\left(\sigma_{I}\right) \\
(\mathrm{mag})\end{array}$ \\
\hline $0.62 \ldots \ldots \ldots \ldots \ldots \ldots$ & 13097 (319) & $14.950(0.019)$ & $14.986(0.015)$ & $14.784(0.021)$ \\
\hline 2.69 & 10991 (308) & $14.875(0.045)$ & $14.788(0.016)$ & $14.343(0.041)$ \\
\hline $3.70 \ldots \ldots \ldots$ & $10579(271)$ & $14.871(0.048)$ & $14.737(0.018)$ & $14.276(0.044)$ \\
\hline $26.62 \ldots \ldots \ldots \ldots \ldots$ & $4976(217)$ & $15.636(0.026)$ & $14.775(0.030)$ & $14.036(0.024)$ \\
\hline $31.61 \ldots$ & $4538(270)$ & $15.798(0.024)$ & $14.822(0.038)$ & $14.053(0.038)$ \\
\hline $34.60 .$. & $4244(166)$ & $15.877(0.021)$ & $14.846(0.035)$ & $14.053(0.035)$ \\
\hline $85.46 \ldots \ldots \ldots \ldots \ldots$ & $2392(126)$ & $16.562(0.050)$ & $15.090(0.027)$ & $14.018(0.035)$ \\
\hline $89.41 \ldots$. & $2222(98)$ & $16.589(0.048)$ & $15.106(0.025)$ & $14.030(0.031)$ \\
\hline $96.62 \ldots \ldots \ldots \ldots \ldots . . . . . . .$. & $2177(55)$ & $16.752(0.040)$ & $15.149(0.024)$ & $14.075(0.030)$ \\
\hline $106.59 \ldots \ldots \ldots \ldots$ & $1888(72)$ & $16.924(0.046)$ & $15.334(0.029)$ & $14.192(0.033)$ \\
\hline $110.55 \ldots \ldots \ldots \ldots . . . . . .$. & $1865(99)$ & $17.119(0.028)$ & $15.449(0.033)$ & $14.270(0.034)$ \\
\hline
\end{tabular}

Note.-Although the data from day 85.46 onward were not used in the EPM analysis, we list the values of the measured parameters through the early part of the transition to the nebular phase for completeness.

${ }^{a}$ Days since discovery, 1999-12-09.82 UT (HJD 2,451,522.32).

which behaves quite differently for the various filter combinations. For the $B V, B V I$, and $V I$ filter combinations $\zeta$ is rather insensitive to color temperature for $T_{c} \gtrsim 8000 \mathrm{~K}$ (see Eastman et al. 1996; Hamuy et al. 2001). As the temperature drops below $8000 \mathrm{~K}, \zeta$ rises for all three filter combinations, and the strength of the temperature dependence varies considerably, from a rather weak dependence in $V I$ to stronger dependencies in $B V I$ and, especially, $B V$.

How might we expect uncertainty in the reddening to affect the derived distances and explosion dates for our data set? Our spectral measurements of SN 1999gi essentially sample two different epochs, one very early $(t<4$ days after discovery) and the other later, during the plateau ( $26<t<35$ days). At the early times the color temperatures derived from all three filter combinations are greater than $8000 \mathrm{~K}$; we therefore expect little change in $\zeta$ for different assumed reddenings at these times. For the later set of data points, however, $T<8000 \mathrm{~K}$, and $\zeta$ will be quite sensitive to reddening changes, especially in the $B V$ and $B V I$ filter combinations. Now, the slope (distance) and $y$-intercept (time of explosion) of the line described by equation (3) are affected somewhat differently by changes in $\theta$. A uniform change in the overall level of $\theta$ at each epoch (i.e., an offset) will only affect the $y$-intercept $\left(t_{0}\right)$. A differential change in $\theta$, on the other hand, will affect both the $y$-intercept and the slope $(D)$. The rapidly changing values of $\zeta$ with $T_{c}$ in the $B V$ and $B V I$ filter combinations should therefore lead to a relatively greater dependence on reddening for the derived distances in these filter combinations than for VI. On the other hand, since all filter combinations are sensitive to the more uniform changes in $\theta$ brought about by changes in $f$ and $B$, we might anticipate the derived explosion times to be more equally affected by reddening uncertainty in the three filter combinations.

The EPM distances and explosion times derived for $B V$, $B V I$, and $V I$ as a function of assumed reddening are shown in Figure 8; to calculate the values, we have limited the EPM analysis to the first six spectral epochs (see discussion below and Fig. 9). To give a sense of the formal uncertainty in the distance for each filter combination, $1 \sigma$ (statistical) error bars sampling reddening increments of $E(B-V)$ $=0.03 \mathrm{mag}$ are also shown. These errors represent the $1 \sigma$ spread in the distances derived for each of the filter combi- nations from 1000 simulated sets of data characterized by the values and uncertainties given in Table 4. As anticipated, distances derived using the $B V$ and $B V I$ filter sets are more sensitive to changes in reddening than are distances determined using the VI filter combination. The convergence of the distances derived using the different filter

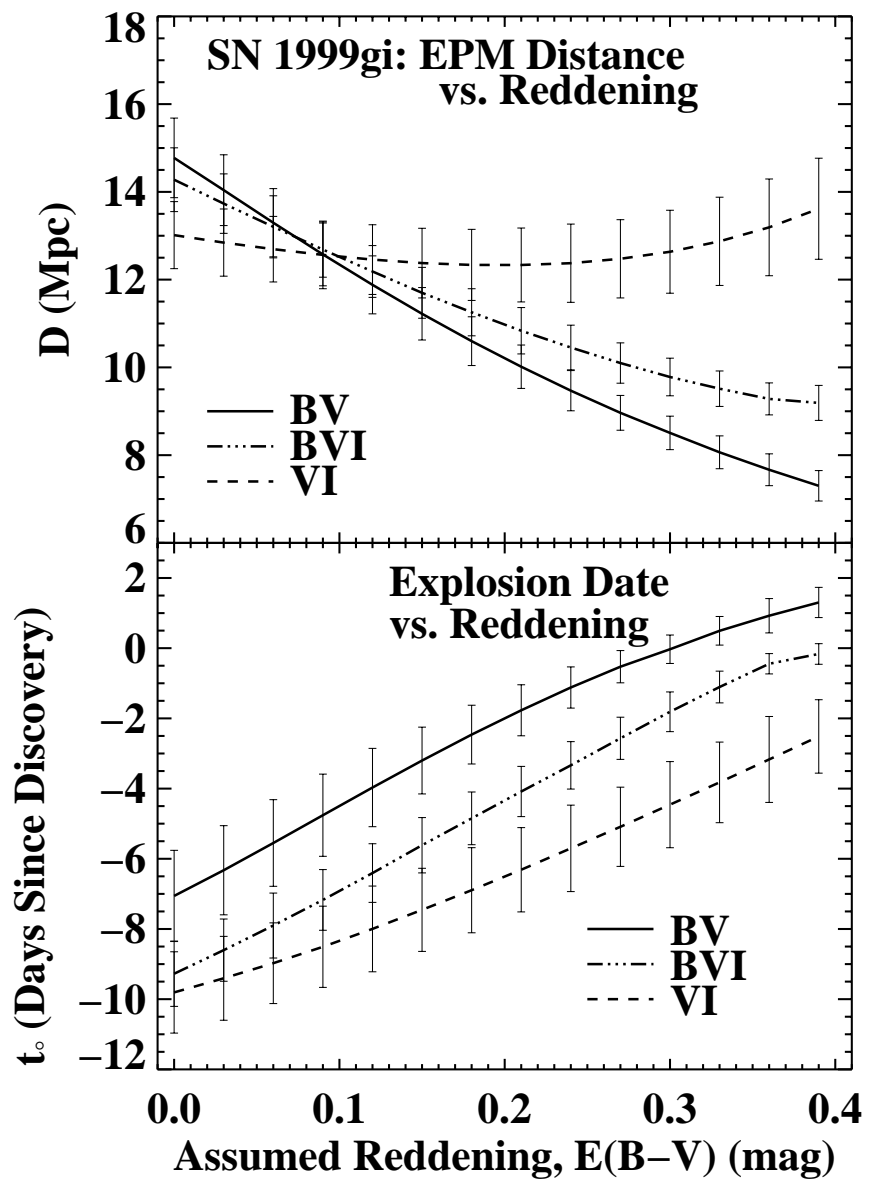

FIG. 8.-Derived EPM distances (top) and explosion times (bottom) as a function of assumed reddening for SN 1999gi using three different filter combinations to estimate the photospheric temperature and flux dilution factor. 


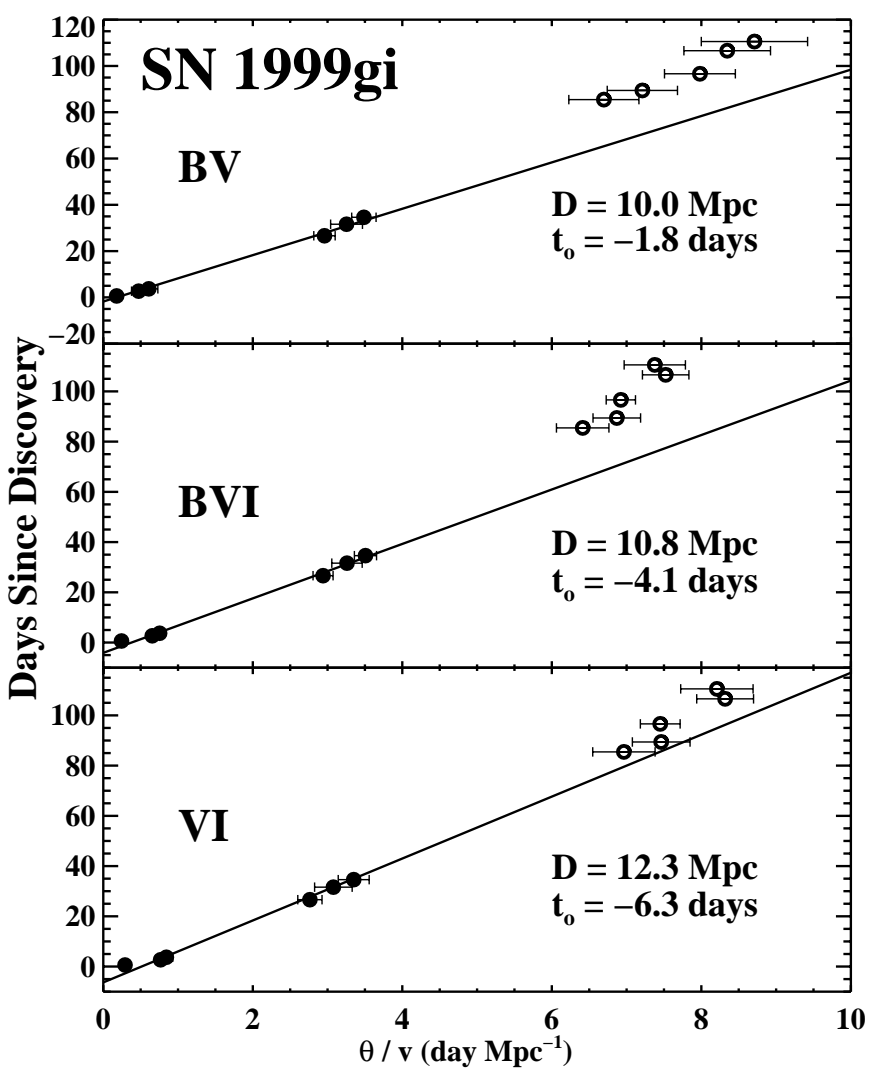

FIG. 9.-Parameters used to derive the distance to SN 1999gi, with photospheric color temperature determined using the $B V(t o p), B V I$ (middle), and VI (bottom) filter combinations; a reddening of $E(B-V)=0.21 \mathrm{mag}$ has been assumed. $\theta$ is the angular size of the optical photosphere (Table 5) and $v$ is the velocity of the gas at the photosphere (Table 4). The line of best fit is determined by applying the criterion of least absolute deviations to the first six epochs (filled circles). The slope of this line yields the distance, $D$, and the $y$-intercept the time of explosion, $t_{0}$. The deviation of the later data points (open circles) from the best-fit line may result from the lack of theoretical models in Eastman et al. (1996) corresponding to the low photospheric temperatures at this late photospheric phase from which to estimate the dilution factor.

combinations at $E(B-V) \approx 0.1 \mathrm{mag}$ agrees with the conclusion of $\S 2.3$ that SN 1999gi suffers little extinction and builds further confidence that the EPM technique itself may be used to estimate SN II-P reddenings (e.g., Hamuy et al. 2001). For the purpose of deriving the final EPM distance and its uncertainty, we shall use the reddening value derived in $\S 2.3, E(B-V)=0.21 \pm 0.09 \mathrm{mag}$, which represents the average of the EPM-derived reddening along with the four other reddening estimates discussed in $\S 2.3$.

The value of $t_{0}$ in Figure 8 also shows somewhat better mutual consistency among the different filter combinations at lower reddenings, but there is no strong preference for a particular reddening value. The inconsistency among the different filter combinations for the derived explosion dates is troubling, and it may result from the use of "average" dilution factors rather than a set custom made for SN 1999gi.

We determine the final distance and time of explosion of SN 1999gi by taking the simple means of the values derived from the three filter combinations for $E(B-V)=0.21 \mathrm{mag}$ and find $D=11.06 \mathrm{Mpc}$ and $t_{0}=4.06$ days before discovery $\left(D=10.02,10.84\right.$, and $12.33 \mathrm{Mpc}$ and $t_{0}=-1.79$, -4.09 , and -6.31 days, for the $B V, B V I$, and $V I$ band- passes, respectively). Although it is difficult to assign a precise statistical error bar to this result, there are two identifiable sources that do contribute quantifiable uncertainty to the values of $D$ and $t_{0}$. First, there is the $1 \sigma$ spread in the values found from the three individual filter combinations for $E(B-V)=0.21 \mathrm{mag}$, which are $\Delta D_{\text {filters }}=1.17 \mathrm{Mpc}$ and $\Delta t_{\text {filters }}=2.26$ days. The second source of uncertainty comes from the uncertainty in the reddening estimate of 0.09 mag. This uncertainty leads directly to an uncertainty in distance of $\Delta D_{\text {reddening }}={ }_{-1.39}^{+1.62} \mathrm{Mpc}$ and an uncertainty in the explosion date of $\Delta t_{\text {reddening }}={ }_{-2.08}^{+1.96}$ days. The final estimate of the statistical uncertainty is found by taking the quadrature sum of the two sources of error. From this, then, our best estimate of the distance and time of explosion of SN 1999gi is $D=11.1_{-1.8}^{+2.0} \mathrm{Mpc}$ and $t_{0}=4.1_{-3.1}^{+3.0}$ days before discovery. Although the uncertainty is somewhat larger than the statistical error associated with the individual distances (especially using the $B V$ and $B V I$ filter combinations), we feel it more accurately reflects the overall uncertainty in the distance, since we have no a priori preference for any of the filter combinations and our reddening estimate suffers from uncertainty as well.

To give an idea of the relevant derived parameters, we list the dilution factor, photospheric angular size, and photospheric color temperature for $E(B-V)=0.21 \mathrm{mag}$ in Table 5 and plot the values used in the EPM analysis for this reddening in Figure 9. In Figure 9 we see that the data obtained near the very end of the plateau ( $t>85$ days; open circles) are somewhat inconsistent with the earlier data; this is probably due to the fact that the theoretical models of Eastman et al. (1996) include no spectra with color temperatures as low as those derived for SN 1999gi at these late times (Table 5), and so the inferred dilution factors rely completely on extrapolation. At these low temperatures the dilution factor, especially in $B V$ and $B V I$, is changing very rapidly with temperature. The EPM distance is therefore more securely obtained from the earlier epochs, and we thus have only used the data from the first six spectral epochs to derive the distance to SN 1999gi.

\section{DISCUSSION}

\subsection{EPM Distance}

The distance to NGC 3184 has previously been estimated through a variety of techniques, and it is useful to compare these with our EPM distance of $D=11.1_{-1.8}^{+2.0} \mathrm{Mpc}$. The earliest estimate is that of de Vaucouleurs (1979), who finds $D=7.9 \mathrm{Mpc}$ from tertiary indicators (diameters, magnitudes, and luminosity index of spiral galaxies). Pierce (1994) derives a Tully-Fisher (TF) distance to NGC 3184 of $D=7.2 \pm 1.7 \mathrm{Mpc}$, although he points out that the low inclination $\left(i=24^{\circ}\right)$ of NGC 3184 makes this distance more uncertain than the statistical error bar alone suggests. Using a similar approach, but with updated models and parameters, Paturel et al. (2002; the result is listed in the LEDA database prior to formal publication) derive $D=11.59$ Mpc. ${ }^{13}$ From the recession velocity corrected for Virgo

13 This distance estimate, given by the MUP parameter in the LEDA database (i.e., using the TF method for spiral galaxies), may change somewhat in the future when a more sophisticated calculation is implemented, which properly accounts for the effects of statistical bias (G. Paturel 2002, private communication). 
TABLE 5

Quantities Derived From the EPM ANaLysis of SN 1999gi

\begin{tabular}{|c|c|c|c|c|c|c|c|c|c|}
\hline DAY ${ }^{b}$ & \multicolumn{3}{|c|}{ Angular Size $\left(10^{8} \mathrm{~km} \mathrm{Mpc}^{-1}\right)^{\mathrm{a}}$} & \multicolumn{3}{|c|}{ DILUTION FACTOR } & \multicolumn{3}{|c|}{ Temperature (K) } \\
\hline $0.62 \ldots \ldots \ldots$ & $1.96(0.31)$ & $2.69(0.17)$ & $3.20(0.28)$ & $0.528(0.027)$ & $0.504(0.009)$ & $0.495(0.010)$ & $32497(5466)$ & $23219(1392)$ & $18964(1515)$ \\
\hline $2.69 \ldots \ldots \ldots$ & $4.25(0.90)$ & $5.94(0.59)$ & $7.00(0.65)$ & $0.421(0.029)$ & $0.426(0.007)$ & $0.439(0.004)$ & $18425(3290)$ & $13619(955)$ & $11284(779)$ \\
\hline $3.70 \ldots \ldots \ldots$ & $5.31(1.12)$ & $6.60(0.65)$ & $7.42(0.73)$ & $0.396(0.024)$ & $0.419(0.006)$ & $0.438(0.004)$ & $15985(2654)$ & $12785(961)$ & $11008(812)$ \\
\hline $26.62 \ldots$. & $13.04(0.27)$ & $12.75(0.20)$ & $11.73(0.48)$ & $0.766(0.053)$ & $0.528(0.009)$ & $0.455(0.007)$ & $5739(190)$ & $6731(98)$ & $7831(295)$ \\
\hline $31.61 \ldots \ldots \ldots$ & $13.07(0.36)$ & $12.92(0.22)$ & $11.93(0.68)$ & $0.936(0.075)$ & $0.571(0.013)$ & $0.461(0.011)$ & $5231(189)$ & $6325(104)$ & $7603(399)$ \\
\hline $89.41 \ldots \ldots \ldots$ & $14.03(0.65)$ & $13.39(0.15)$ & $14.35(0.38)$ & $2.026(0.146)$ & $0.943(0.033)$ & $0.564(0.018)$ & $3770(112)$ & $4716(84)$ & $5889(165)$ \\
\hline $96.62 \ldots \ldots \ldots$ & $15.15(0.78)$ & $13.21(0.18)$ & $14.05(0.37)$ & $2.351(0.128)$ & $1.013(0.033)$ & $0.563(0.017)$ & $3547(78)$ & $4554(73)$ & $5898(160)$ \\
\hline $106.59 \ldots \ldots$ & $13.75(0.76)$ & $12.45(0.14)$ & $13.62(0.36)$ & $2.315(0.144)$ & $1.050(0.039)$ & $0.595(0.022)$ & $3570(92)$ & 4475 (79) & $5621(169)$ \\
\hline $110.55 \ldots \ldots$ & $14.12(0.85)$ & $12.06(0.21)$ & $13.29(0.36)$ & $2.537(0.119)$ & $1.129(0.029)$ & $0.614(0.024)$ & $3437(66)$ & $4323(51)$ & $5482(170)$ \\
\hline
\end{tabular}

Note.- Values shown here assume a reddening of $E(B V)=0.21 \mathrm{mag}$. Although the data from day 85.46 onward were not used to determine the EPM distance, we list the values of the measured parameters through the early part of the transition to the nebular phase for completeness. Errors are $1 \sigma$ (statistical), derived from 1000 sets of artificial data characterized by the uncertainties listed in Table 4 . Note that unrounded values and uncertainties are given for all variables to aid reproduction of the analysis carried out in this paper.

a Angular size of the optical photosphere (i.e., the surface of last scattering).

b Days since discovery, 1999-12-09.82 UT (HJD 2,451,522.32).

infall (Bottinelli et al. 1986; Sandage \& Tammann 1990) and assuming $H_{0}=75 \mathrm{~km} \mathrm{~s}^{-1} \mathrm{Mpc}^{-1}$, LEDA also lists a kinematic distance of $D=10.09 \mathrm{Mpc}$. Finally, although relatively isolated, NGC 3184 is cataloged to be in a small group of four galaxies (Tully 1988), two of which have had Cepheid distances derived by the HST Key Project (see Freedman et al. 2001 and references therein). The final Key Project distances to these two possible group-member galaxies, corrected for metallicity, are $D=13.30 \pm 0.55 \mathrm{Mpc}$ (NGC 3319) and $D=13.80 \pm 0.51 \mathrm{Mpc}$ (NGC 3198).

The distance estimates to NGC 3184 (or galaxies that may be in its group) therefore span a range $7.2 \lesssim D \lesssim 13.8$ Mpc. Our EPM distance of $11.1_{-1.8}^{+2.0} \mathrm{Mpc}$ is certainly consistent with this range, and finds best agreement with the updated TF and kinematic distances listed in LEDA. We note, however, that the EPM distance is somewhat inconsistent with the two Cepheid group-member distances in the sense $D_{\text {EPM }}<D_{\text {Cepheid }}$ by $\sim 20 \%$ for the average of the two Cepheid distances. Since the uncertainty inherent in comparing distances derived among putative group-member galaxies could certainly account for much of the discrepancy, it is not clear how meaningful this comparison is. Along these lines we point out that the direct comparison between a Cepheid distance to a host galaxy of an SN II-P (the type for which EPM is most securely applied) has only been carried out thus far for one object, SN 1973R (see L02 for a complete listing of Cepheid-EPM distance comparisons), and, unfortunately, the EPM distance is highly uncertain $(\sim 50 \%)$. The $H S T$ Cycle 10 program to directly compare the Cepheid-based distance to the host galaxy of SN 1999em (Leonard et al. 2002b) with the EPM distances derived by L02 and Hamuy et al. (2001) will certainly offer one solid critical test of the consistency of these two primary extragalactic distance indicators. Given the importance of firmly establishing the value of $H_{0}$ from primary distance indicators, additional direct comparisons between the Cepheid distances of the host galaxies of well-observed SNe II-P and the EPM distances seem warranted.

Knowing the distance to SN 1999gi allows us to compare its absolute brightness with that of previous SNe II-P. For nine photometrically confirmed SNe II-P with EPM distances, L02 find the mean plateau absolute magnitude to be $\langle M\rangle_{V}($ plateau $)=-16.4 \pm 0.6 \mathrm{mag}$, with SN $1999 \mathrm{em}$ itself having $\langle M\rangle_{V}$ (plateau) $=-15.9 \pm 0.2 \mathrm{mag}$. Correcting for $A_{V}=0.65$ mag (i.e., adopting $E(B-V)=0.21 \mathrm{mag}$ and $R_{V}=3.1$, Savage \& Mathis 1979), we measure $\langle M\rangle_{V}($ plateau $)=-16.0 \pm 0.4 \mathrm{mag}$ for SN 1999gi, where the uncertainty incorporates both the uncertainty in the EPM distance and the uncertainty in the reddening. Again, the similarity between SN 1999gi and SN 1999em argues that these were very similar events. The consistency of $\langle M\rangle_{V}$ (plateau) for SN 1999gi with previous SNe II-P also strengthens the suggestion by L02 that distances good to $\sim 30 \%(1 \sigma)$ may be possible for SNe II-P by simply measuring $\langle m\rangle_{V}$ (plateau), obviating the need for a complete EPM analysis unless a more accurate distance is desired. ${ }^{14}$

\subsection{Progenitor Mass}

Recently, S01 and S02 utilized a relatively new technique to set limits on the progenitor masses of core-collapse SNe. Essentially, the procedure is as follows. (1) Search preexplosion images of the SN's host galaxy (either ground-based or, preferably, from $H S T$ ) to look for a progenitor star at the location of the SN (the use of postexplosion HST images is especially helpful here to pinpoint the exact SN location). (2) If no progenitor is found, determine the detection threshold of the image. (3) Assume a distance to the galaxy and a reddening to the SN (and assume it is the same as the reddening to the progenitor star) and use the appropriate bolometric corrections to translate the detection threshold into an upper bound on the progenitor luminosity. (4) Use $\mathrm{H}$ II region studies of the host galaxy to establish a likely metallicity for the progenitor star. (5) Use stellar evolution models to predict the final preexplosion luminosity and effective temperature of stars spanning the range of initial

\footnotetext{
${ }^{14}$ Hamuy \& Pinto (2002) refine this technique, showing that a measurement of the plateau magnitude and the ejecta expansion velocity potentially yields a considerably smaller uncertainty in the derived distance.
} 
masses believed to result in core-collapse $\mathrm{SNe}$ of the observed type (e.g., for SNe II-P resulting from metallicity $Z=0.04$ stars S01 eliminate all progenitors with $M>25$ $M_{\odot}$, since such stars are expected to undergo significant mass loss, which is deemed to be inconsistent with the observed characteristics of SNe II-P). Finally, (6) determine which progenitor masses would have remained undetected given the detection threshold of the image. Since more massive main-sequence stars generally result in brighter progenitors, it may be possible to set an upper mass limit for an undetected progenitor star through this approach.

The first of two objects examined by these studies is SN 1999gi (the other is SN 1999em), for which two prediscovery HST WFPC2 images of NGC 3184 exist, one taken through the F606W filter (central wavelength $5957 \AA$ ) for $160 \mathrm{~s}$ and the other through the F300W filter (central wavelength 2911 $\AA$ ) for $800 \mathrm{~s}$. Using two postexplosion $H S T$ images to pinpoint the SN position, $\mathrm{S} 01$ are unable to detect a progenitor star in either image. From simulations with synthetic stars, S01 derive the image detection limits as well as the uncertainty in these limits. To turn this into a luminosity limit, they then assume a distance to NGC 3184 of $D=7.9 \mathrm{Mpc}$. This distance is adopted due to the general agreement that they found among three estimates: (1) the kinematic distance using the Strauss et al. (1992) recession velocity of 592 $\mathrm{km} \mathrm{s}^{-1}$ and $H_{0}=75 \mathrm{~km} \mathrm{~s}^{-1} \mathrm{Mpc}^{-1}$, with no correction for Virgo infall (yields $D=7.9 \mathrm{Mpc}$ ); (2) the TF distance derived by Pierce (1994, $D=7.2 \pm 1.7 \mathrm{Mpc}$ ); and (3) the distance estimated by tertiary indicators (de Vaucouleurs $1979, D=7.9 \mathrm{Mpc})$. From their analysis of the colors of the stars in the $\mathrm{OB}$ association, to which the progenitor of SN 1999gi presumably belonged, S01 conclude that $E(B-V) \approx 0.15 \mathrm{mag}$, in good agreement with the values found by our other estimates $(\S 2.3)$. The $\mathrm{H}$ II region study of NGC 3184 by Zaritsky et al. (1994) leads S01 and S02 to conclude that the cluster containing SN 1999gi has a metallicity somewhere between solar and twice solar, and so they adopt $Z=0.04$ for the progenitor. They then use the Geneva evolutionary tracks (Meynet et al. 1994; Schaller et al. 1992), which follow stars up to the point of core carbon ignition, to estimate the preexplosion luminosity of progenitor stars for 7-40 $M_{\odot}$. Comparing the progenitor star luminosities with the image detection threshold, then, leads S01 to set an upper limit for the progenitor of SN 1999gi of $9_{-2}^{+3} M_{\odot}$.

In a similar analysis, but for the progenitor of $\mathrm{SN}$ 1999em, S02 introduce an improved stellar evolutionary model based on the most recent version of the Eggleton (1971, 1972, 1973) evolution program, which follows stars all the way through their carbon-burning lifetimes. It is shown that the final luminosities predicted for progenitor stars of 7-12 $M_{\odot}$ are significantly affected by the carbonburning stage, curiously predicting stars of 7-10 $M_{\odot}$ to be more luminous in their final stages than those with 11$15 M_{\odot}$. Despite these changes in the evolutionary code, S02 state that a reanalysis of the SN 1999gi data using the new stellar models results in the same upper limit as that previously derived. Since stars less massive than $\sim 8$ $10 M_{\odot}$ are not expected to undergo core collapse, an upper mass limit of $9_{-2}^{+3} M_{\odot}$ for the progenitor of SN $1999 \mathrm{gi}$ is highly restrictive, and it narrows the range of possible progenitor masses to be essentially between 8 and $12 M_{\odot}$. Interestingly, for SN $1999 \mathrm{em}, \mathrm{S} 02$ find it very difficult to reconcile the lack of a detection with the theoretically pre- dicted final luminosities of the progenitor stars: given the stated limits, all stars with initial mass greater than $7 M_{\odot}$ should have been detected. It is only by considering the uncertainty on the threshold limit $(\sim 0.2$ dex $)$ that S02 are able to (barely) explain the nondetection and then only for a very narrow range of possible progenitor masses, $12 \pm 1 M_{\odot}$.

We now reexamine the progenitor mass limit of $\mathrm{SN}$ 1999gi in light of our present study. We note that the distance adopted to NGC 3184 by S01 is significantly shorter than the more recently derived distances $(\S 4.1)$, including our EPM distance to SN 1999gi of $D=11.1_{-1.8}^{+2.0} \mathrm{Mpc}(\S 3)$. Naturally, a longer distance will result in a brighter intrinsic luminosity detection threshold. Reproducing the analysis of S01, Figure 10 shows the revised detection limits using $D=11.1 \mathrm{Mpc}$ and $E(B-V)=0.21 \mathrm{mag}$ for the progenitor of SN 1999gi, along with the Geneva evolutionary tracks of 7-40 $M_{\odot}$ stars for $Z=0.04$ metallicity. From this analysis, the upper mass limit for a nondetection of the progenitor star for SN 1999gi increases to $15_{-3}^{+5} M_{\odot}$; following S01, the uncertainty is simply set by the nearest modeled progenitor masses on either side of the observed limit.

Using the more recent stellar evolutionary models of S02 (which are only explicitly done for $Z=0.02$ metallicity, though metallicity changes do not substantially alter the

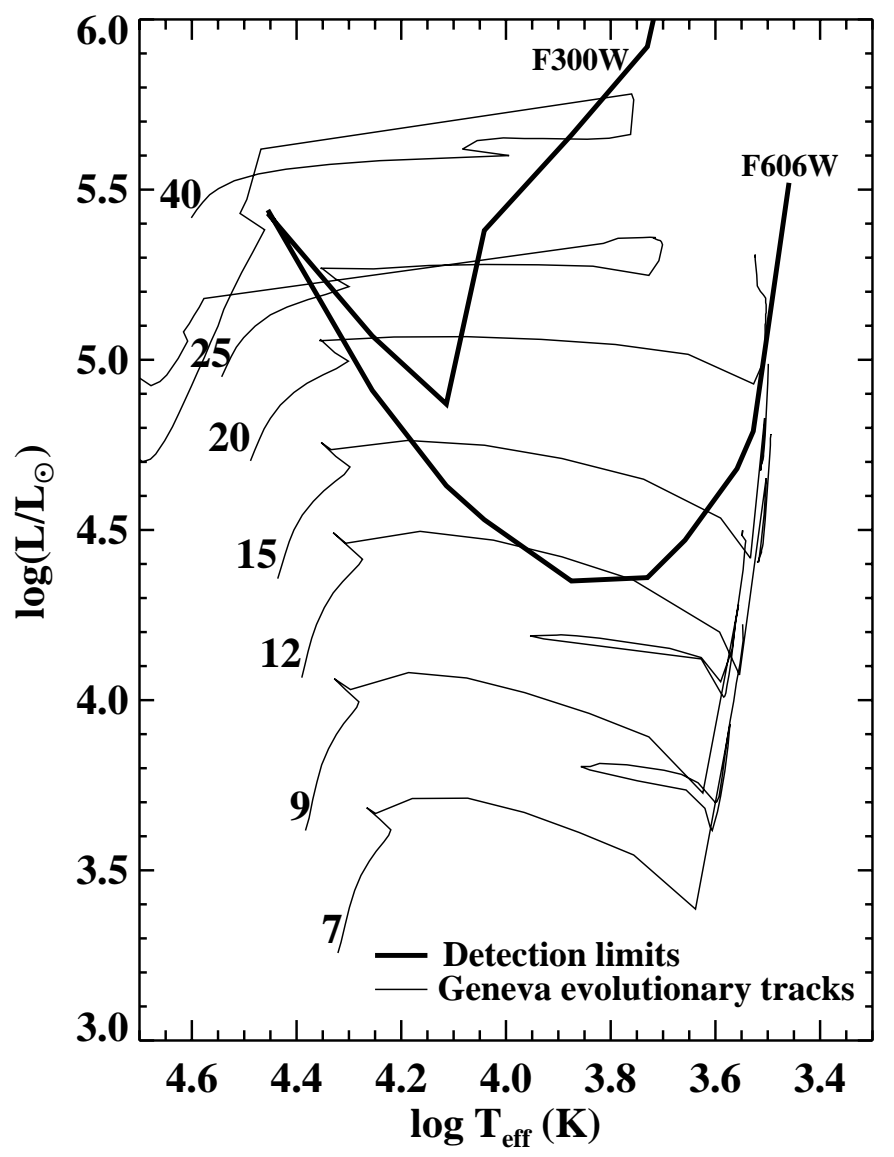

FIG. 10.-Geneva evolutionary tracks (Meynet et al. 1994; Schaller et al. 1992) for 7-40 $M_{\odot}$ stars (thin lines) plotted with the lower detection limit of the prediscovery image taken with the F300W and F606W filters (thick lines) with $H S T$ as determined by $\mathrm{S} 01$ and modified to reflect the EPM distance and reddening to SN 1999gi derived in this work. Using these models, the nondetection of the progenitor star implies an upper mass limit of $15_{-3}^{+5} M_{\odot}$ for the progenitor. 


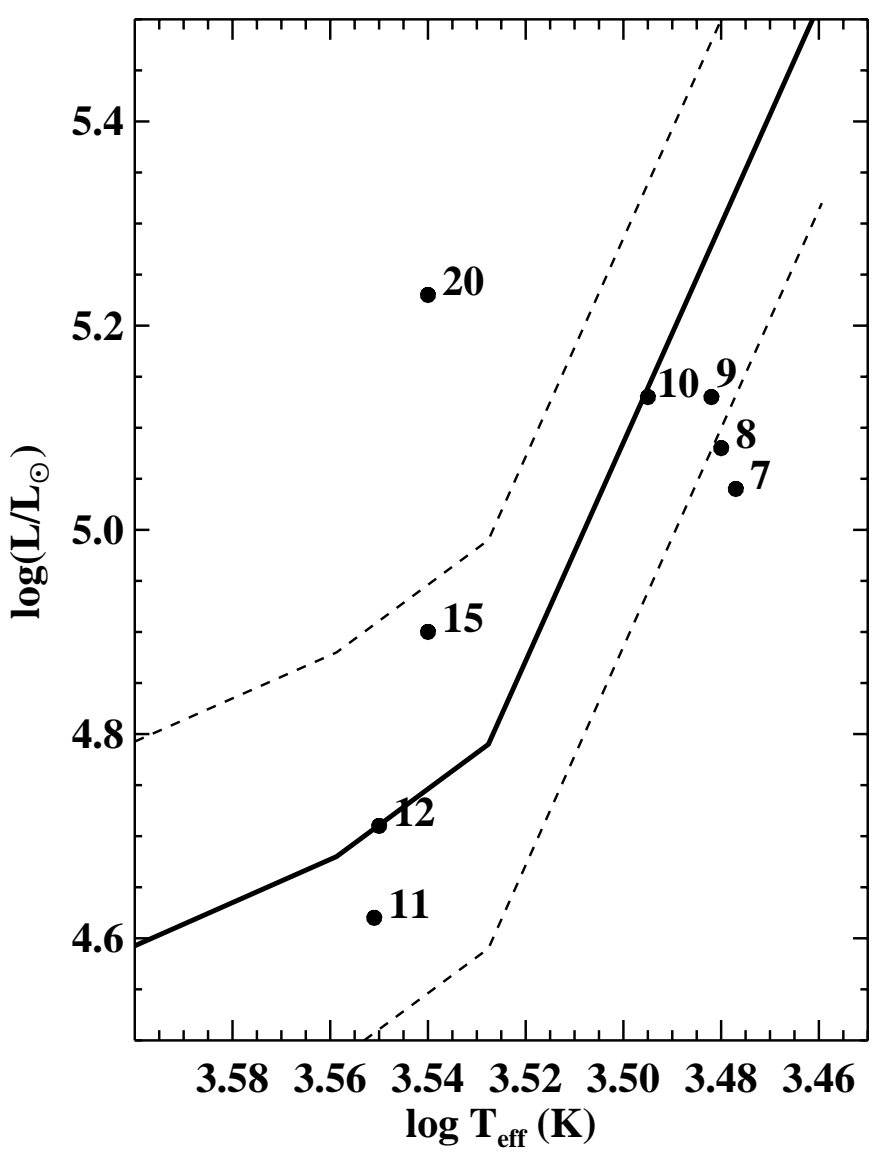

FIG. 11.-Close-up of the final evolutionary state for stars of 7-20 $M_{\odot}$, using the updated models of S02. Also shown (thick solid line) is the detection limit of the F606W filter HST WFPC2 observation of NGC 3184, as well as the upper and lower range of the uncertainty in the detection limits (dashed lines). Applying the same criteria as S02 results in an upper bound on the progenitor mass of $15_{-3}^{+5} M_{\odot}$.

final preexplosion luminosity at the low masses considered), Figure 11 shows a close-up of the expected preexplosion position for stars of 7-20 $M_{\odot}$ along with the detection limits of the F606W filter; the uncertainties in the detection limits (taken to be 0.2 dex) are also shown. From this we see that only progenitors with masses greater than $\sim 12 M_{\odot}$ should have been detected. Moreover, when we consider the uncertainty in the detection threshold itself, as was done by $\mathrm{S} 02$, it is possible to include all progenitors $\leq 15 M_{\odot}$ among those that could conceivably have avoided detection. Applying the same criterion as the most recent analysis by $\mathrm{S} 02$, then, results in an updated upper mass limit for the progenitor of SN 1999gi of $15_{-3}^{+5} M_{\odot}$.

Hence, using the techniques employed by S01 and S02, but using our revised distance and reddening estimates, we arrive at a consistent value of $15_{-3}^{+5} M_{\odot}$ as the upper mass limit for the progenitor of SN 1999gi, compared with the upper limit of $9_{-2}^{+3}$ - derived by S01 and S02. The increased upper limit results mainly from the larger distance derived through the EPM than was assumed by the earlier analyses. It is unfortunate that the only three progenitor stars thus far positively identified for Type II supernovae are for unusual events $(\S 1)$. Clearly, the detection of a progenitor star in a preexplosion image for an SN II-P would have much to tell us about the late stages of stellar evolution for isolated massive stars, and would serve as a very useful check on the abil- ity of models to accurately predict their final luminosity. However, since the uncertainty in the detection limits for SN 1999gi (and SN 1999em as well) is of the same order as the theoretically derived luminosity differences among the candidate progenitor stars, the ability of the Smartt et al. technique to robustly discriminate among progenitors in the range $7-15 M_{\odot}$ is questionable.

\section{CONCLUSIONS}

We present 15 optical spectra and 30 photometric epochs of SN 1999gi sampling the first 169 and 174 days since discovery, respectively, and derive its EPM distance. Our main conclusions are as follows.

1. SN 1999gi is a Type II-P event with a photometric plateau lasting until about 100 days after discovery. It reached $B$ maximum on 1999 December $13.7 \pm 1.8$ (HJD $2,451,526.2 \pm 1.8$, or $3.9 \pm 1.8$ days after discovery), and achieved peak $B$ and $V$ magnitudes of $\sim 14.8$ and $\sim 14.6$, respectively. Overall, we find the photometric behavior of SN 1999gi to be extremely similar to that of SN $1999 \mathrm{em}$.

2. The very early-time spectra of SN 1999gi confirm the existence of the high-velocity absorption features in the profiles of $\mathrm{H} \beta$ and $\mathrm{He}$ I $\lambda 5876$ that were first identified by Baron et al. (2000) in spectra of SN 1999em. The highest-velocity feature $\left(\mathrm{H} \beta\right.$, day 1) extends out to nearly $-30,000 \mathrm{~km} \mathrm{~s}^{-1}$, implying the existence of very high velocity material in the outer envelope of SN 1999gi at early times. These features are verified to be true P Cygni profiles, consisting of both an absorption trough and an emission peak in early-time spectra. The high-velocity features are not seen, however, in $\mathrm{H} \alpha$ at early times.

3. By comparing the early-time spectral shape with blackbody functions we derive an upper limit on the reddening of SN 1999gi of $E(B-V)<0.45$ mag; comparison with the color evolution of SN 1999em suggests an even lower limit, of $E(B-V)<0.30 \mathrm{mag}$. Other reddening estimates are consistent with these limits, and imply a somewhat lower reddening, $E(B-V)=0.21 \pm 0.09 \mathrm{mag}$, which we adopt as the preferred reddening value.

4. Our best estimate for the EPM distance and explosion time of SN 1999gi is $D=11.1_{-1.8}^{+2.0} \mathrm{Mpc}$ and $t_{0}=4.1_{-3.1}^{+3.0}$ days prior to discovery. This distance is consistent with some recent distance estimates to NGC 3184 , but is 20\% shorter than the average of the Cepheid distances derived to two putative group-member galaxies.

5. The EPM distance implies an average plateau brightness of $\langle M\rangle_{V}$ (plateau $)=-16.0 \pm 0.4 \mathrm{mag}$, which is very similar to the value found for SN 1999em and consistent with the average plateau brightness found by L02 of $\langle M\rangle_{V}($ plateau $)=-16.4 \pm 0.6$ mag for 9 photometrically confirmed SNe II-P with EPM distances.

6. Following the analysis methods of S01 and S02 we derive a new upper mass limit for the progenitor of $\mathrm{SN}$ 1999gi of $15_{-3}^{+5} M_{\odot}$, which is substantially less restrictive than the original limit of $9_{-2}^{+3} M_{\odot}$ found by S01 and S02. The higher limit comes mainly from the longer distance derived through the EPM than was assumed by the earlier analyses.

We thank Alison Coil and Maryam Modjaz for assistance with the observations, and Georges Paturel for a helpful correspondence regarding the LEDA database (http:// leda.univ-lyon1.fr), of which we made use. An anonymous 
referee made suggestions that resulted in an improved manuscript. We are also grateful to the Lick Observatory and FLWO staffs for their support of the telescopes. This research has made use of the NASA/IPAC Extragalactic Database, which is operated by the Jet Propulsion Laboratory, California Institute of Technology, under contract with NASA. Our work was supported in part by NASA through the American Astronomical Society's Small Research Grant Program and by grants AR-8754, GO8648, GO-9114, and GO-9155 from the Space Telescope Science Institute, which is operated by the Association of
Universities for Research in Astronomy, Inc., under NASA contract NAS 5-26555. Supernova research at the Harvard/ Smithsonian Center for Astrophysics is supported by NSF grant AST 98-19825. Additional funding was provided to A. V. F. by NASA/Chandra grant GO-0-1001C, by NSF grant AST 99-87438, by the Guggenheim Foundation, and by the Sylvia and Jim Katzman Foundation. KAIT was made possible by generous donations from Sun Microsystems, Inc., the Hewlett-Packard Company, AutoScope Corporation, Lick Observatory, the NSF, the University of California, and the Katzman Foundation.
Aldering, G., Humphreys, R. M., \& Richmond, M. 1994, AJ, 107, 662 Barbon, R., Benetti, S., Cappellaro, E., Rosino, L., \& Turatto, M. 1990, A\&A, 237, 79

Baron, E., et al. 2000, ApJ, 545, 444

Baron, E., Hauschildt, P. H., \& Branch, D. 1994, ApJ, 426, 334

Baron, E., et al. 1995, ApJ, 441, 170

Baron, E., Hauschildt, P. H., Branch, D., Wagner, R. M., Austin, S. J., Filippenko, A. V., \& Matheson, T. 1993, ApJ, 416, L21

Bessell, M. S. 1999, PASP, 111, 1426

Bottinelli, L., Gouguenheim, L., Paturel, G., \& Teerikorpi, P. 1986, A\&A, 156,157

Cohen, J. G., Darling, J., \& Porter, A. 1995, AJ, 110, 308

Cohen, M. H. 1996, The LRIS Polarimeter

Cousins, A. W. J. 1981, South African Astron. Obs. Circ., 6, 4

de Vaucouleurs, G. 1979, ApJ, 227, 729

Eastman, R. G., \& Kirshner, R. P. 1989, ApJ, 347, 771

Eastman, R. G., \& Pinto, P. A. 1993, ApJ, 412, 73

Eastman, R. G., Schmidt, B. P., \& Kirshner, R. 1996, ApJ, 466, 911

Eggleton, P. P. 1971, MNRAS, 151, 351

. 1972, MNRAS, 156, 361 1973, MNRAS, 163, 279

Fabricant, D., Cheimets, P., Caldwell, N., \& Geary, J. 1998, PASP, 110, 79

Filippenko, A. V. 1982, PASP, 94, 715

.1988, AJ, 96, 1941

1993, IAU Circ. 5737

. 1997, ARA\&A, 35, 309

Filippenko, A. V., Barth, A. J., Bower, G. C., Ho, L. C., Stringfellow, G. S., Goodrich, R. W., \& Porter, A. C. 1995, AJ, 110, 2261 (Erratum: 1996, 112, 806)

Filippenko, A. V., Li, W. D., Treffers, R. R., \& Modjaz, M. 2001, in ASP Conf. Ser. 246, Small Telescope Astronomy on Global Scales, ed W. P. Chen, C. Lemme, \& B. Paczyński (San Francisco: ASP), 121

Filippenko, A. V., Porter, A. C., Sargent, W. L. W., \& Schneider, D. P. 1986, AJ, 92, 1341

Freedman, W. L., et al. 2001, ApJ, 553, 47

Gibson, B. K. 2000, Mem. Soc. Astron. Italiana, 71, 693

Goodrich, R. W., Stringfellow, G. S., Penrod, G. D., \& Filippenko, A. V. 1989, ApJ, 342, 908

Hamuy, M. 2001, Ph.D. thesis, Univ. Arizona

Hamuy, M., et al. 2001, ApJ, 558, 615

Hamuy, M., \& Pinto, P. A. 2002, ApJ, 566, L63

Höflich, P. 1991, A\&A, 246, 481

Horne, K. 1986, PASP, 98, 609

Johnson, H. L., Mitchell, R. I., Iriarte, B., \& Wisniewski, W. Z. 1966, Commun. Lunar Plate. Lab., 4, 99

Kirshner, R. P., \& Kwan, J. 1974, ApJ, 193, 27

Landolt, A. U. 1992, AJ, 104, 340

Lejuene, T., \& Schaerer, D. 2001, A\&A, 366, 538

Leonard, D. C., \& Filippenko, A. V. 2001, PASP, 113, 920

Leonard, D. C., Filippenko, A. V., Ardila, D. R., \& Brotherton, M. S. 2001, ApJ, 553, 861

Leonard, D. C., et al. 2002a, PASP, 114, 35 (L02)

\section{REERENCES}

Leonard, D. C., et al. 2002b, in preparation

Massey, P., \& Gronwall, C. 1990, ApJ, 358, 344

Matheson, T., Filippenko, A. V., Ho, L. C., Barth, A. J., \& Leonard, D. C. 2000, AJ, 120, 1499

Meynet, G., Maeder, A., Schaller, G., Schaerer, D., \& Charbonnel, C. 1994, A\&AS, 103, 97

Miller, J. S., \& Stone, R. P. S. 1993, Lick Obs. Tech. Rep., 66

Mitchell, R. C., Baron, E., Branch, D., Hauschildt, P. H., Nugent, P. E., Lundqvist, P., Blinnikov, S., \& Pun, C. S. J. 2002, ApJ, 574, 293

Montes, M. J., \& Wagoner, R. V. 1995, ApJ, 445, 828

Munari, U., \& Zwitter, T. 1997, A\&A, 318, 269

Nakano, S., et al. 1999, IAU Circ. 7329

Oke, J. B., et al. 1995, PASP, 107, 375

Oke, J. B., \& Gunn J. E. 1983, ApJ, 266, 713

Patat, F., Barbon, R., Cappellaro, E., \& Turatto, M. 1994, A\&A, 282, 731

Paturel, G., et al. 2002, in preparation

Pierce, M. J. 1994, ApJ, 430, 53

Press, W. H., Teukolsky, S. A., Vetterling, W. T., \& Flannery, B. P. 1992 Numerical Recipes in C (2d ed.; Cambridge: Cambridge Univ. Press)

Richmond, M. W., et al. 1995, AJ, 109, 2121

Ryder, S., Staveley-Smith, L., Dopita, M., Petre, R., Colbert, E., Malin, D., \& Schlegel, R. 1993, ApJ, 416, 167

Sandage, A., \& Tammann, G. 1990, ApJ, 365, 1

Savage, B. D., \& Mathis, J. S. 1979, ARA\&A, 17, 73

Schaller, G., Schaerer, D., Meynet, G., \& Maeder, A. 1992, A\&AS, 96, 269

Schlegel, D. J., Finkbeiner, D. P., \& Davis, M. 1998, ApJ, 500, 525

Schmidt, B. P. 1993, Ph.D. thesis, Harvard Univ.

Schmidt, B. P., Kirshner, R. P., \& Eastman, R. G. 1992, ApJ, 395, 366

Schmidt, B. P., et al. 1993, Nature, 364, 600

Smartt, S. J., Gilmore, G. F., Tout, C. A., \& Hodgkin, S. T. 2002, ApJ, 565 1089 (S02)

Smartt, S. J., Gilmore, G. F., Trentham, N., Tout, C. A., \& Frayn, C. M. 2001, ApJ, 556, L29 (S01)

Stone, R. P. S. 1977, ApJ, 218, 767

Strauss, M. A., Huchra, J. P., Davis, M., Yahil, A., Fisher, K. B., \& Tonry, J. 1992, ApJS, 83, 29

Szentgyorgyim A. H., et al. 2002, in preparation

Trondal, O., Granslo, B. H., Kushida, R., Nakano, S., Yoshida, S., \& Kadota, K. 1999, IAU Circ. 7334

Tully, B. 1988, Nearby Galaxies Catalog (Cambridge: Cambridge Univ. Press)

Van Dyk, S. D., Filippenko, A. V., \& Li, W. 2002, PASP, 114, 700

Van Dyk, S. D., Peng, C. Y., Barth, A. J., \& Filippenko, A. V. 1999a, AJ, 118,2331

Van Dyk, S. D., et al. 1999b, PASP, 111, 313

Wade, R. A., \& Horne, K. 1988, ApJ, 324, 411

Walborn, N. R., Lasker, B. M., Laidler, V. G., \& Chu, Y. H. 1987, ApJ, 321, L41

White, G. L., \& Malin, D. F. 1987, Nature, 327, 36

Woosley, S. E., \& Weaver, T. A. 1986, ARA\&A, 24, 205

Yadav, R. K. S., \& Sagar, R. 2001, MNRAS, 328, 370

Zaritsky, D., Kennicutt, R. A., \& Huchra, J. P. 1994, ApJ, 420, 87 